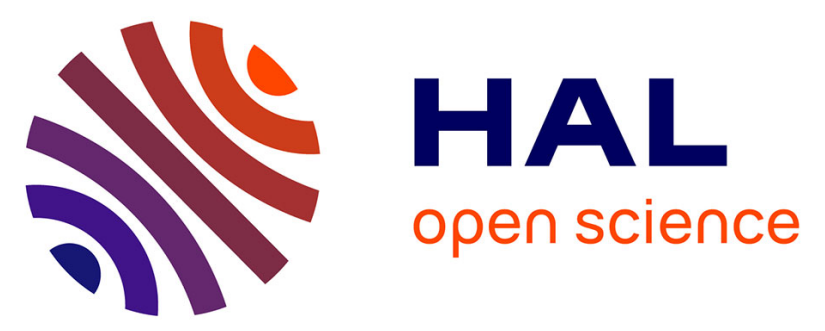

\title{
Advances in element speciation analysis of biomedical samples using synchrotron-based techniques
}

Francesco Porcaro, Stéphane Roudeau, Asunción Carmona, Richard Ortega

\section{To cite this version:}

Francesco Porcaro, Stéphane Roudeau, Asunción Carmona, Richard Ortega. Advances in element speciation analysis of biomedical samples using synchrotron-based techniques. Trends in Analytical Chemistry, 2018, 104, pp.22-41. 10.1016/j.trac.2017.09.016 . hal-01905442

\section{HAL Id: hal-01905442 \\ https://hal.science/hal-01905442}

Submitted on 18 Dec 2018

HAL is a multi-disciplinary open access archive for the deposit and dissemination of scientific research documents, whether they are published or not. The documents may come from teaching and research institutions in France or abroad, or from public or private research centers.
L'archive ouverte pluridisciplinaire HAL, est destinée au dépôt et à la diffusion de documents scientifiques de niveau recherche, publiés ou non, émanant des établissements d'enseignement et de recherche français ou étrangers, des laboratoires publics ou privés. 
Advances in element speciation analysis of biomedical samples using synchrotron-based techniques

Francesco Porcaro ${ }^{a, b}$, Stéphane Roudeau ${ }^{a, b}$, Asuncion Carmona ${ }^{a, b}$, Richard Ortega ${ }^{a, b, *}$

a University of Bordeaux, CENBG, UMR 5797, F-33170 Gradignan, France.

${ }^{\mathrm{b}} \mathrm{CNRS}$, IN2P3, CENBG, UMR 5797, F-33170 Gradignan, France.

* Corresponding author. Tel: +33 557120907

E-mail address: ortega@cenbg.in2p3.fr (R. Ortega)

\title{
Highlights
}

- $\quad$ principle of direct element speciation with synchrotron X-ray absorption spectroscopy (XAS)

- experimental modalities for bulk- and micro-XAS speciation and their limitations

- review (2012-2017) of XAS in pharmacology, metals and nanoparticles toxicology, physiopathology

- future directions and developments of XAS speciation for biomedical research

\begin{abstract}
Synchrotron-radiation X-ray absorption spectroscopy (XAS) is a direct method for speciation analysis with atomic resolution, providing information about the local chemical environment of the probed element. This article gives an overview of the basic principles of XAS and its application to element speciation in biomedical research. The basic principle and experimental modalities of XAS are introduced, followed by a discussion of both its limitations, such as beam damage or detection limits, and practical advices to improve experiments. An updated review of biomedical studies involving XAS published over the last 5 years is then provided, paying special attention to metal-based drug biotransformation, metal and nanoparticle toxicology, and element speciation in cancer, neurological, and general pathophysiology. Finally, trends and future developments such as hyphenated methods, in situ correlative imaging and speciation, in vivo X-ray Absorption Near Edge Spectroscopy (XANES), full-field XANES, and X-ray Free Electron Laser (XFEL) XAS are presented.
\end{abstract}

\section{Keywords}

synchrotron; XAS; speciation; EXAFS; XANES; XFEL; metals ; nanoparticles; cancer, neuropathology. 


\section{Introduction}

The IUPAC (International Union of Pure and Applied Chemistry) definition of speciation in chemistry is; 'The distribution of an element amongst defined chemical species in a system', and the definition for speciation analysis is; 'Analytical activities of identifying and/or measuring the quantities of one or more individual chemical species in a sample' [1]. In the field of speciation analysis, X-ray absorption spectroscopy (XAS) is a singular and powerful method with an increasing number of applications in biomedical research. The uniqueness of XAS compared with other analytical methods for speciation derives from its atomic resolution [2-4]. XAS is based on the measurement of the modulation of X-ray absorption by an atom around the core-level binding energies. XAS probes the core level electrons in an element specific manner, it is sensitive to the oxidation state, coordination chemistry, and the distances, coordination number and species of the atoms immediately surrounding the selected element. XAS is therefore a powerful tool for matter investigation in different scientific fields, from catalysis and geology to bioinorganic chemistry. The high sensitivity of XAS, with limits of detection in the tens of $\mu \mathrm{g} \cdot \mathrm{g}^{-1}$ range or $\mathrm{mM}$ range, makes it applicable to trace element speciation in biology, where it can be performed on solids or liquids without the need for complex sample preparation. Accordingly, this review will focus only on XAS element speciation of biomedical samples.

To begin, the physical principle of XAS, the distinction between XANES (X-ray Absorption Near Edge Spectroscopy) and EXAFS (Extended X-ray Absorption Fine Structure), the various modalities of operation from bulk XAS to spatially resolved micro-XAS, and the strengths and limitations of the techniques with a special attention to biological samples analysis will be presented. Then XAS applications in various fields of biomedical research will be reviewed, over the last five years, to give an overview of the state of the art and the trends in this domain. For works published in previous periods the reader may refer to several valuable review articles [5-9]. This review will be limited to the application of XAS on biomedical samples, but interesting examples of XAS element speciation of bioenvironmental samples such as plants or micro-organisms can be found in other recent articles [1013]. Due to the simultaneous measurement of many important parameters, XAS is among the best techniques for determining the "structure-to-function" relationship in metalloprotein studies. XAS structural studies of metal-binding sites from purified metalloproteins, which are often carried out jointly with X-ray crystallography, will not be considered in this review, information about this procedure can be found in other articles [14-16]. However, the emerging development of XAS in combination with chromatographic methods to characterize proteins from complex samples will be discussed.

This review will cover XAS element speciation in the fields of metal-based pharmacological compounds, metal and nanoparticle (NP) toxicology, trace element physiology, and trace element dyshomeostasis in the etiology of cancer, neurological disorders and other diseases. The aim of this article is to highlight which biomedical questions have been recently addressed by using XAS, and how this has been achieved.

\section{Principle and modalities}

\subsection{Principle of XAS}

From a phenomenological point of view, XAS arises from the photo-electric effect, resulting from the absorption of an X-ray photon by an electron in a bound quantum core level (i.e. from 1s or $2 p$ levels) of an atom. Each core electron has a well-defined quantum binding energy, characteristic of the element. When the incident $\mathrm{X}$-rays are scanned across one of these energies, there is an abrupt increase in absorption. Such feature in the spectrum is the so-called 'absorption edge' sometimes also referred as 'white line', at an energy characteristic of the element (Figure 1). The electron may be 
removed from its quantum level only if the incident X-ray energy is higher than the binding energy of the electron. Photon energies in the X-ray regime are required to excite core level electrons with typical binding energies ranging from about $100 \mathrm{eV}$, for the lower $Z$ elements, to about $100 \mathrm{keV}$ for the higher $\mathrm{Z}$ elements. The absorption coefficient $(\mu \mathrm{E})$ gives the probability that $\mathrm{X}$-rays will be absorbed according to Lambert-Beer's law (Eq. 1).

$$
I=I_{0} e^{-\mu(E) x} \quad \text { (Eq. 1) }
$$

where $\mathrm{I}$ is the $\mathrm{X}$-ray intensity transmitted through the sample, $\mathrm{I}_{0}$ is the incident intensity, and $\mathrm{x}$ the sample thickness.

XAS is generally performed at synchrotron radiation facilities where intense and tunable monochromatic X-ray beams are achievable. Beyond its element specificity, XAS can be performed on diluted samples, a fundamental feature for biological studies. XAS is usually carried out by measuring the photons transmitted through the sample by monitoring the incident and transmitted photon flux with photodiodes. However, for diluted analytes such as trace elements in biological samples, XAS must be carried out in X-ray fluorescence mode, a more sensitive detection mode. The X-ray fluorescence mode consists in placing an X-ray detector, usually a solid-state multi-detector at $90^{\circ}$ with respect to the incoming beam. In X-ray fluorescence mode, the absorption coefficient is measured as a function of the $X$-ray fluorescence yield $I_{f}$ normalized by $I_{0}$ (Eq. 2 ):

$$
\mu(E) \propto I_{f} / I_{0} \quad \text { (Eq. 2) }
$$

In a typical XAS spectrum two peculiar regions can be recognized due to the different kind of information provided, as reported in Figure 1. The region in the energy range of -50 to $+200 \mathrm{eV}$ with respect to the edge position provides information primarily about geometry and oxidation state of the probed element and its analysis is defined as X-ray absorption near edge structure spectroscopy (XANES). A second region in the energy range of +200 to $+1000 \mathrm{eV}$ with respect to the edge position provides information about metal site ligation and bond distances. The study of such region is the core of the extended X-ray absorption fine structure spectroscopy (EXAFS).

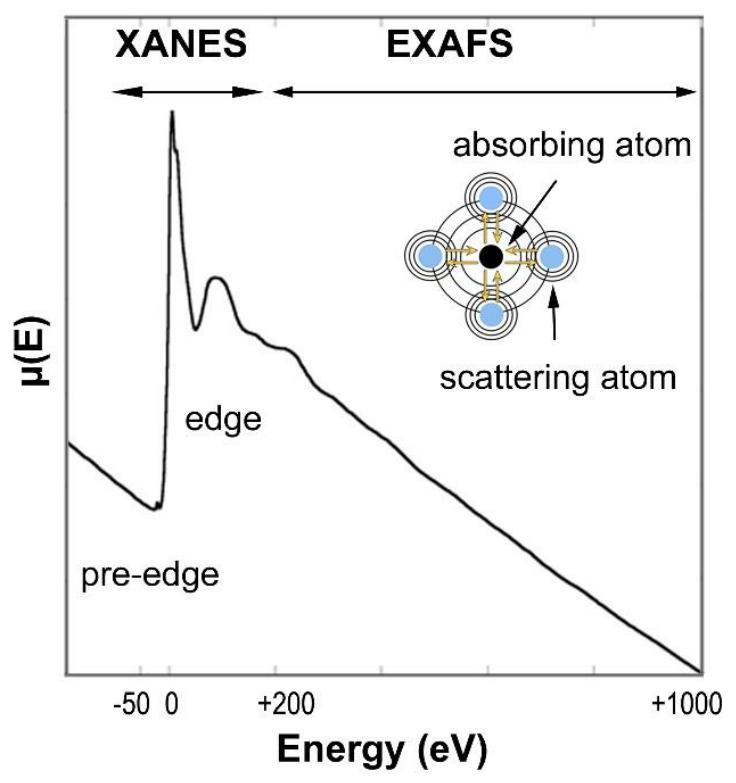

Fig. 1. X-ray absorption spectroscopy. Schematic representation of a XAS spectrum showing the characteristic variation of the absorption coefficient $\mu(E)$ in function of the $X$-ray incident energy. The sharp rise in $\mu(E)$ at the absorption edge energy, the oscillations of $\mu(E)$ after the edge, as well as the XANES and EXAFS regions are schematically depicted. Adapted with permission from [6]. 
An accurate mathematical description of XAS is beyond the scope of this review but a detailed explanation of XAS principle can be found in several excellent monographs [2-4]. Thereafter, we will provide the basics equations in order to offer the necessary tools to better comprehend XAS advantages and drawbacks in the field of chemical speciation analysis. A large difference exists between EXAFS and XANES data analysis. Indeed, since EXAFS theory has been studied for almost 40 years, a solid and elegant semi-classical and quantum theory has been developed. The EXAFS signal, as reported in a pictorial and simplified description in Figure 1, can be approximated to the interference pattern generated by photoelectron waves travelling from the absorber atom to the proximity scattering atoms, and back. The scattered photo-electron can return to the absorbing atom, modulating the amplitude of the photo-electron wave. This in turn modulates the absorption coefficient $\mu(E)$ causing the EXAFS. As a consequence, thanks to wave theory, an equation can be written down describing the behavior of the photoelectron with wave number $k$, created and propagating away from the atom. Therefore, the EXAFS equation reported below as a function of the fine structure $\chi$ (Eq. 3) according to [2], allows for a full quantitative approach analysis of the EXAFS spectrum:

$$
\begin{aligned}
& \chi_{t h}(k)=S_{0}^{2} \sum_{i}\left[\frac{N_{i} A_{i}(k)}{k R_{i}^{2}}\right] \cdot \sin \left[\left(2 k R_{i}+\phi_{i}(k)\right] e^{\left(-2 \sigma_{i}^{2} k^{2}\right)} e^{\left[\frac{-2 R_{i}}{\lambda_{i}(k)}\right]}(\text { Eq. 3) }\right. \\
& \mathrm{R}_{\mathrm{i}}=\mathrm{R}_{0 \mathrm{i}}+\Delta \mathrm{R}_{\mathrm{i}} \\
& \mathrm{k}=\sqrt{\frac{2 m_{e}}{\hbar^{2}}\left(E-E_{0}\right)}
\end{aligned}
$$

The EXAFS equation is the sum of the contribution of each scattering atom type, where $i$ represents the individual coordination shell of identical atoms at approximately the same distance from the central absorber atom. Although Eq. 3 is formally valid for single scattering processes, i.e. photoelectrons travelling just back and forth from the absorber to a single proximal atom, this equation can also be applied for describing multiple scattering phenomena in which the photoelectron travels through many additional scattering pathways before coming back to the absorber. As reported by Kelly and coworkers [17], the terms $A(k), \Phi(k)$, and $\lambda(k)$ are the effective scattering amplitude, the phase shift, and the mean free path of the single photoelectron, respectively, all of which can be simulated by computer programs. $\mathrm{S}_{0}{ }^{2}$ is a constant parameter defined as the passive electron reduction factor and takes into account for the slight relaxation of the remaining electrons in the presence of the core hole vacated by the photoelectron. The term $\sigma^{2}$, namely the Debye-Waller factor, accounts for the disorder in the interatomic distances and can represent the mean-square displacement of the bond length between the absorber atom and the coordination atoms in a shell. The term $R_{i}$ (Eq. 4 ) is the photoelectron half path length i.e., the distance between the absorber and a coordinating atom for a single-scattering event. The term $\Delta \mathrm{R}_{\mathrm{i}}$ justifies the change of the interatomic distance relative to the initial path length Ri. Noticeably, equation (Eq. 5) is used to express the kinetics energy of the photoelectron in wavenumber, $k$, by using the mass of the electron $m_{e}$ and the reduced Plank's constant $\hbar$. The proper selection of EO during the data treatment define the correct energy scale of the photoelectron and justify the mismatch between the energy scales of the theoretical spectrum compared to the experimental one. Finally, researchers are usually interested in the remaining variables, determined by modeling the EXAFS spectrum: the type of atom related to $A(k)$, the coordination number $\mathrm{N}$ and distance $\mathrm{R}$. 
The fitting and modeling of signal can be performed with a variety of different software. A nonexhaustive list can be found at: http://www.esrf.eu/UsersAndScience/Experiments/TBS/SciSoft/Links/xafswebsite.

Concerning the theoretical simulation, a number of $a b$ initio codes allows for reproduction or even fittings of the XANES region of absorption spectra such as FEFF [18], FDMNES [19], and MXAN [20]. A detailed guide to EXAFS data analysis is beyond the scope of present review but the reader is strongly advised to consult more specific materials on the topic $[3,17]$. Briefly, data processing can be slit up in two main parts; 1) pre-treatment of experimental data to extract the EXAFS signal, 2) model refinement using a "ratio method" or nonlinear least squares fitting of data by means of empirical or theoretical standards. The pretreatment itself also consists of a multistep procedure; correction for instrumental effects (self-absorption, monochromator "glitches" removal), merging of different sample scans and normalization of the obtained average spectrum, extraction of EXAFS oscillations (selection of the energy threshold EO, subtraction of smooth background typically using polynomial functions) and Fourier transformation. It is important to stress that EXAFS signal consists of a sum of sine waves with an amplitude that depends on the wavenumber $(k)$. As a consequence, EXAFS spectrum Fourier transformation into $\mathrm{R}$ space is necessary to analyze the atomic sine waves to obtain the atomic coordination. Importantly, since the amplitude of EXAFS oscillations decreases with increasing energy above the absorption edge, EXAFS spectra are consequently k-weighted by $\mathrm{k}$ raised to the power of 1, 2, or 3 before the Fourier transform filtering.

Compared to EXAFS, there is not a simple physical description of XANES and no XANES equation is available for accurate quantitative analysis of the signal. As a consequence several strategies have been developed for XANES interpretation. For example, besides computer simulation based on multiple scattering or Density Function Theory already cited [18-20], it is possible to obtain reliable semi-quantitative analysis by comparing different spectra from a set of well-known reference samples. Then, mathematical tools such as the linear combination fitting (LCF) of the measured reference samples and a statistical treatment with Principal Component Analysis (PCA) can be exploited to determine chemical speciation of the absorber in a quick and relatively easy way. However, it is important to keep in mind that XAS always provides an average picture of the local atoms environment. In the XANES part of the spectrum the selected element edge is very sensitive to energy shift or shape alterations caused by element oxidation state, geometry or ligands nature modifications. Despite this, mathematical tools such as the LCA of measured reference samples and the statistical treatment with PCA allows a semi-quantitative refinement of empirical edge observations in XANES.

\subsection{XAS modalities}

XAS requires a high flux of incident X-rays and therefore is developed almost exclusively on dedicated beamlines at synchrotron radiation facilities. As a direct speciation analysis method XAS can be performed in situ with minimal sample preparation. XAS of biomedical samples is most often applied ex vivo on biological tissues and fluids, and in vitro on cultured cells. In vivo analyses are usually not possible because of the intense irradiation damage induced by the X-ray beam, although some examples of in vivo speciation have been reported, see section 4.3.

As previously mentioned, XAS is quite arbitrary segmented into two energy regions, XANES and EXAFS that provide complementary information on the speciation of a selected element. These two methods also differ in their domain of application since EXAFS will generally require a higher element concentration in samples than XANES. The EXAFS description of the fine oscillations of the absorption coefficient after the edge is more demanding in terms of signal intensity than the simple determination of the absorption edge energy by XANES. Because elements of interest are usually quite diluted in biomedical samples, XANES is more easily performed. However this is a general assumption since in 
some cases, for example when pre-edge structures of the XANES region are studied, XANES will require higher concentrations of the elements. When XAS is applied with a micro- or a nano-beam the amount of elements are usually low within the sample volume probed by the focused beam, which again limits the applicability of EXAFS compared to XANES. With focused beams on biological samples, XANES is the most widely used method. Micro-EXAFS can however be performed when the element concentrations are locally high.

The most usual modalities for XAS element speciation of biomedical samples are summarized in Figure 2. Bulk XAS analysis is performed with unfocused beams, giving an average information on the chemical species present in the probed volume. Bulk XAS is a very useful approach to quantify the proportion of different chemical species of an element on a large and therefore representative sample volume. One of the drawbacks of bulk XAS is that information generated about all the chemical species present is mixed. To palliate this lack of selectivity two complementary approaches can be carried out. First, to combine XAS to a chromatographic method in order to separate the distinct molecules containing the element of interest, as reviewed in section 4.1. The other possible approach is the use of focused beams to probe chemical species in selected regions of the sample. This microscopic speciation can be attained by several means as commented below and schematized in Figure 2. For microscopic speciation modes, elemental density maps are almost always generated by synchrotron $\mathrm{X}$-ray fluorescence microscopy (micro-SXRF) in order to select regions of interest. XAS micro-speciation can also be combined with other microscopic characterization in a correlative manner and will be reviewed in section 4.2 .

Micro-XAS in point analysis mode is the most widely employed micro-speciation modality. Point micro-XAS involves the acquisition of an XAS spectrum on a single spot of the sample by keeping the sample static under the micro-beam, and scanning the energy edge of the element. This strategy is often applied since it can be done quickly and provides information about the chemical species in specific regions of the samples. The spatial resolution of point micro-XAS will vary in function of the beamline focusing optics but it usually ranges from few hundred of nanometers to few microns. The main drawbacks of point micro-XAS are that it can induce beam damage such as photo-reduction due to long and intense irradiation of the same region (see section 2.5.), and that only a few regions of the sample are analyzed which might not be representative of the full sample. An alternative to point micro-XAS analysis is the chemical specific XRF imaging where a XRF image of the chemical species are obtained at specific energies. Chemical specific XRF imaging offers great advantages compared to micro-XANES point analysis, it results in 2D images and therefore it is not restricted to a limited number of points in the image. Chemical specific XRF imaging requires shorter times of beam irradiation greatly limiting the potential photo-chemical artifacts. However, chemical specific XRF imaging is only possible for chemical species that are well resolved in the XANES spectrum. It is for instance possible to map selenium chemical species [21], $\mathrm{Cr}(\mathrm{III})$ vs $\mathrm{Cr}(\mathrm{VI})$ oxidation states [22], arsenic chemical species [23-24], and sulfur chemical species [25]. A third alternative for microscopic speciation analysis is micro-XANES imaging that consists in recording a full XANES spectrum for each pixel of the image. Micro-XANES imaging is time consuming since it requires moving the sample in 2 dimensions and acquiring energy scans for each sample position. It has been rarely applied to biological samples since it would need prohibitive acquisition times due to the low concentrations of the elements. This drawback could be overcome in principle using a 2D photon detector, referred to as full-field XANES imaging (FF XANES), then the horizontal and vertical scanning can be avoided using an unfocused beam, which greatly reduces the acquisition times. Again, the limitation of FF XANES imaging is the sensitivity of the 2D detectors which still make this method difficult to apply to biological samples as discussed in section 4.4. 


\section{Bulk}

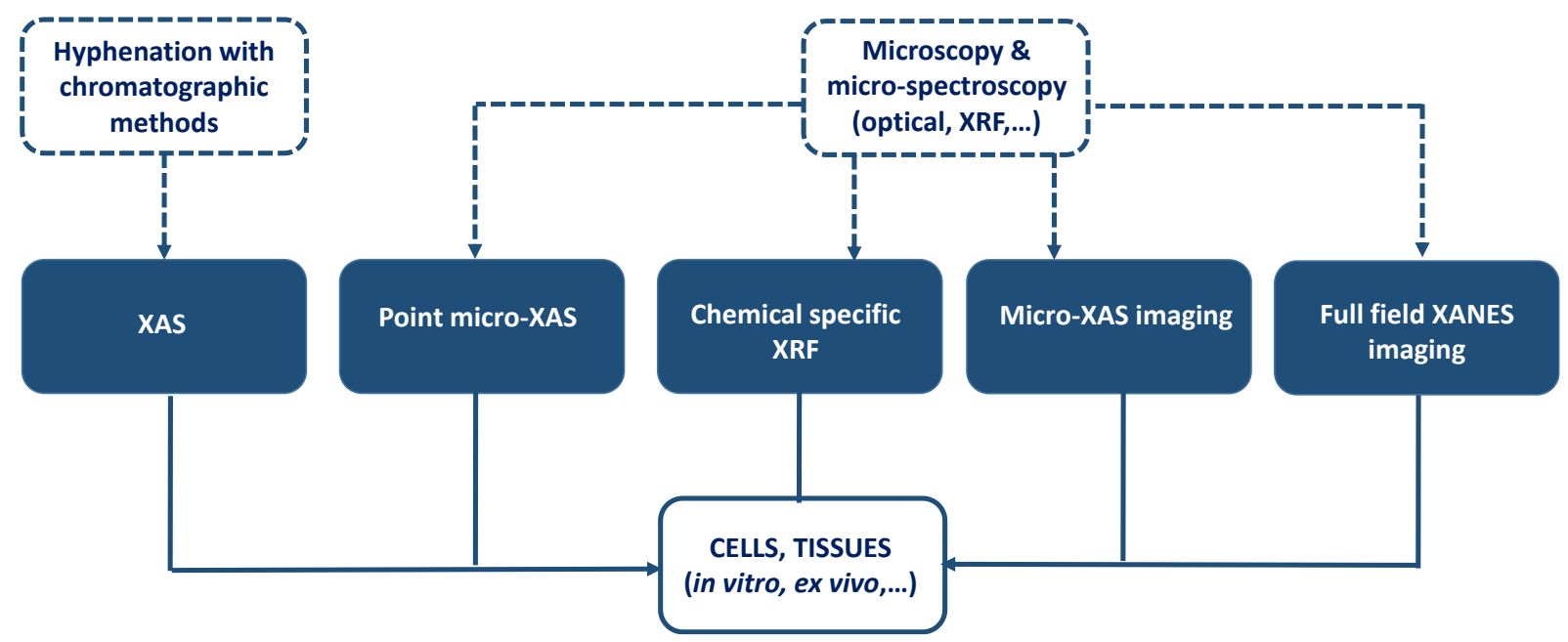

Fig. 2. XAS modalities (full lines) and potential combinations with other methods (dotted lines). Bulk and spatially resolved speciation can be performed using XAS. Bulk speciation can be carried out directly or after chromatographic separation of the analytes to improve selectivity. At least four modes of synchrotron-based microscopic speciation have been developed, point analysis micro-XAS being the most widely employed. Microscopic XAS modes can be advantageously combined to other microscopic techniques such as optical microscopy or XRF, enabling correlative microscopy and microspectroscopy.

\subsection{Standards for $X A S$}

The analysis of reference compounds is first required for the energy calibration of the experiment. A thin foil of the element of interest is usually scanned across the absorption edge energy. As it will be illustrated in various examples in section 3, one important parameter in order to successfully exploit XAS data is the use of XAS reference spectra. Especially in the case of XANES, which has many applications for the speciation of diluted elements in biological systems, the data treatment often consists in fitting a complex experimental spectrum by the linear sum of individual spectra from pure compounds using LCF. Thin metal foils and pure compounds spectra are available from internet sources such as from the University of Chicago (http://cars.uchicago.edu/xaslib/search), or from the International X-ray Absorption Society portal (http://ixs.iit.edu/database/). In addition, it can be useful for speciation purposes to analyze certified reference materials of specific chemical species to monitor the validity of the analytical process. Certified reference compounds of specific chemical species are available for elements such as $\mathrm{As}, \mathrm{Cr}, \mathrm{Hg}$, $\mathrm{Sn}$, Se and $\mathrm{Pb}$. A table of the commercially available certified reference materials useful for speciation analysis can be consulted from the EVISA, European Virtual Institute for Speciation Analysis, website.

(http://www.speciation.net/Public/Document/2005/11/18/CRMs for Speciation.html).

\subsection{Limit Of Speciation (LOS)}

In a previous report we defined the limit of speciation (LOS) for XAS as corresponding to 10 times the standard deviation of XAS blank measurements [26], in analogy to the limit of quantification 
(LOQ) as defined by Currie [27]. The LOS for XAS is highly dependent on the experimental setup of the synchrotron radiation beamline, which will depend on multiple parameters; the incident photon flux and the detection system amongst them. For example the LOS for micro-XANES at arsenic K-edge absorption energy on ID22 beamline at ESRF was calculated to be as low as $13 \mu \mathrm{g} \cdot \mathrm{g}^{-1}$ [26]. Another example to illustrate XAS LOS is given by the bulk EXAFS experiments at $\mathrm{Zn}$ and $\mathrm{Cu}$ K-edges on FAME beamline at ESRF that could be performed on proteins separated by isoelectric focusing with about 10 $\mu \mathrm{g} \cdot \mathrm{g}^{-1}$ of elements [28]. Limits of speciation down to $1 \mu \mathrm{g} \cdot \mathrm{g}^{-1}$ in XANES mode and down to $10 \mu \mathrm{g} \cdot \mathrm{g}^{-1}$ in EXAFS mode have already been reported on the recently developed FAME-UHD beamline at ESRF, using high energy resolution fluorescence detection XAS (HERFD-XAS) for structural investigation of ultra-diluted samples [29]. These values give an idea of the LOS that can be reached with the most sensitive instrumental conditions. It is important to consider that the speciation of elements present in concentrations close to the LOS will result in long acquisition times and poor signal-to-noise ratios that could limit the exploitation of XAS data, i.e. for XANES pre-edge structures or EXAFS data analysis. Therefore bulk XANES and EXAFS are usually carried out on solid samples with element concentrations around $10-100 \mu \mathrm{g} \cdot \mathrm{g}^{-1}$. For micro-XAS another parameter is also important, the thickness of the sample. In order to perform micro-XAS speciation samples are usually thin, i.e. tissue sections or single cells of few microns, thinner than the volume that can be probed by the X-ray beam when applied on bulk samples. Finally, LOS will strongly depend on each experimental setup as discussed. The feasibility of XAS in terms of LOS and element concentration should be discussed prior to the experiment with the beamline scientists of the dedicated facility.

\subsection{Preservation of the native chemical species}

Although XAS is a direct method of speciation that requires minimum sample processing some artifacts in the analytical procedure could compromise the validity of the results. However, if proper procedures are employed, XAS has the capability to probe chemical species close to their native state. Two major bias can be encountered during XAS speciation of biological samples. First is the modification of the native chemical species during sample processing, and second the alteration of chemical species during XAS analysis, due to beam irradiation damage.

A systematic study of the effect of sample preparation on sulfur speciation from rat brain sections was performed by Hackett et al. [30]. They compared frozen unfixed tissues to air dried, freeze dried and formaldehyde-fixed tissues by XANES at S K-edge. The study showed that the formaldehyde fixation had an important impact on the chemical speciation of sulfur with a large increase of disulfide and sulfoxide species, and the decrease of thiols and disappearance of sulfinic and sulfonic acid species, which were already close to the LOS. Air drying and freeze drying protocols resulted in similar contributions of sulfur speciation, comparable to the ones obtained from frozen hydrated tissues. In air dried and freeze dried tissues, a small increase of disulfide and sulfoxide species and the disappearance of the small initial quantity of sulfinic acid were noted. This study highlights the changes in sulfur speciation triggered by the aldehyde fixation. Chemical fixation with aldehydes has previously been shown to modify trace metal concentrations in biological samples [31-33]. Due to its invasive nature, chemical fixation should be avoided for sample preparation of XAS experiments.

XAS is often applied to tissue biopsies or to cultured cells. In both cases samples must be extracted from their initial substrate, the living organisms or the culture dishes, and as it is now generally adopted for many other analytical methods in biology, cryogenic sampling is the method of choice to preserve both chemical and morphological structures [34]. Rapid freezing just after sampling can be performed by a variety of cryogenic protocols such as plunge freezing, slam freezing and high pressure freezing. In any case, the use of cryogenic conditions for sampling followed by the storage in 
an inert atmosphere all along the analytical process is highly advocated to preserve the initial chemical species [26]. Keeping the sample at low temperature also during the analysis offers the supplementary advantage to limit the irradiation damage and to reduce the element loss that is induced by intense beam irradiation, especially when a focused beam is used to perform micro-XAS.

This fully cryogenic protocol requires the availability of a sample cryostage on the XAS beamline, which is most often the case. However in some cases sample cryostages are not available, then samples must be dried and special attention must be paid to check that the chemical species are not modified. One positive effect of drying is that the formation of free radicals from the hydrolysis of water during irradiation can be avoided reducing the beam damage. However drying the samples can affect the speciation of the element of interest, i.e. when a labile water molecule binds to a metal. If bulk analysis is to be performed then air drying is usually performed since no morphological information on the tissue is expected. If micro-XAS has to be performed, then biological samples are usually freeze dried after rapid freezing to better maintain the morphological structure of the tissues or cells [32]. An alternative to drying consists in performing XANES in vivo on hydrated samples at room temperature but has severe limitations due to beam damage. This analytical approach is discussed in section 4.3.

The second main reason for chemical species modification is due to the photo-chemical reactions induced by the X-ray beam on the elements [35]. This is particularly the case when high photon fluxes are delivered, a condition often required to perform XAS on biological samples because elements are present in low concentrations. The situation is even worsened when using X-ray focusing optics to perform micro-XAS compared to bulk XAS due to the high photon density [36]. Photoreduction consecutive to the photo-ionization process is the main artifact induced by intense X-ray beams, although more rarely photo-oxidation can also occur for some specific elements such as sulfur and arsenic [30,37]. To avoid changes of chemical species during X-ray beam irradiation, several strategies can be employed. The delivered dose might be minimized first by choosing an optimal X-ray flux density, second by using cryogenic conditions of analysis. Reducing the delivered dose can be either achieved by reducing the X-ray flux density to the minimal value required to obtain adequate signal-to-noise ratios, and/or by reducing the irradiation time, this late can be optimized for example using high solid angle detectors. As previously discussed, cryogenic XAS is recommended to maintain the native chemical species in the sample, but also to minimize the effects of beam damage and photoreduction $[26,35]$. In practice there are several tricks to minimize the photo-reduction, for example it is better recommended to perform several rapid energy scans on different regions of the sample, by moving the sample of about the beam size between each scan, than to perform a single energy scan on the same sample area which will require long dwell times. Once the experimental design has been optimized the absence of photo-reduction effects must be evaluated. This can be checked by rapid XAS scans on a reference compounds, using the same beam conditions as the ones to be used for the real samples. It must further be verified on the real samples by following the reproducibility of the acquired XAS spectra during time with several consecutive energy scans.

\section{Fields of application}

\subsection{Speciation of metal-based pharmacological compounds}

In the field of inorganic medicinal compounds, owing to its outstanding analytical strength, XAS represents an important tool to unravel the fate of drugs in cells and tissues. Additionally, micro-SXRF imaging can be used to monitor the intracellular localization of a drug as it moves through specific pathways within biological environments. Such information can be acquired at different stages during the movement of a drug through a bio-environment, enabling the detection of potential drug 
interactions with other biological molecules. As a consequence, XAS can definitively boost our knowledge concerning the stability of inorganic and bioinorganic drugs, their uptake and therapeutic mechanism of action [7, 38]. Many examples of XAS exploitation emerge from studies involving metalbased anticancer compounds, such as platinum, ruthenium, gallium, or vanadium metal-complexes.

Due to their greater kinetic inertness, Pt(IV) complexes are extensively studied for cancer treatment as an alternative to the more toxic and cancer resistance inducer Pt(II) drugs. Although an evident edge energy shift in the XAS spectrum between the two oxidation states, it is difficult to accurately determine the $\mathrm{Pt}(\mathrm{IV}) / \mathrm{Pt}(\mathrm{II})$ proportion using standard curve fitting techniques. In this context, Hall and coworkers reported that the white line height of XANES spectrum of Pt complexes, normalized to the post-edge minima (Figure 3), can be used to quantitatively determine the Pt(IV) reduction amount in a mixture [39]. The method allowed for the correlation of Pt(IV) complexes reduction over time to their reduction potentials and cytotoxicity both in cell growth media and A2780 cells. Moreover, such a study confirmed the proposed "bioreductive activation" mechanism of Pt compounds observed indirectly by means of other techniques. This XAS approach for Pt oxidation state assessment was strengthened by comparative results obtained by Chen and collaborators on DLD-1 cancer cells [40]. However, the correlation between edge position and oxidation state is far from being so straightforward. As already mentioned, the energy edge position is also affected by the ligand nature. Indeed, the proposed Hall model does not take the binding of sulfur atoms to Pt into account, which is responsible for a shift to lower energy of the metal edge. Further examples of tricky edge energy shift correlations are illustrated by studies of ruthenium compounds.

Hummer and collaborators applied XAS spectroscopy and micro-SXRF to investigate in vitro and ex vivo $\mathrm{Ru}(\mathrm{III})$ complexes with anticancer activity [41]. Remarkably, the measured edge energy position from a XAS starting database of Ru standard was reported as a function of the theoretical coordination charge calculated according to Allred-Rochow scale. The obtained curve showed a linear correlation used as basis for the coordination/oxidation states assignment in solution and tissue spectra. Despite the precise and well reported methodology, both edge calibration curve and XANES analysis relying on LCF and PCA approaches failed in revealing a prevalent Ru oxidation state in vitro and ex vivo. Levina and coworkers reported a correlation in vitro between the energy edge decrease and changes in averaged $\mathrm{Ru}$-ligands bond lengths consistent with formation of $\mathrm{Ru}(\mathrm{III})-\mathrm{S}$ shorter bonds [42]. In a recent paper by Gransbury and collaborators, a new XAS investigation on Ru compounds in tumor-mimetic environments stressed once again that a strict correlation between the number of coordinated $\mathrm{S} / \mathrm{Cl}$ ligands and energy edge position, impairs the precise oxidation state determination and renders distinction of exact coordination spheres difficult [43]. Although the precise Ru oxidation state is still to be elucidated, XAS techniques provided other fundamental information about Ru compounds. First of all, micro-SXRF studies confirmed a high penetration depth of Ru-based drugs into the closer packed tissue regions and an interesting correlation with iron bio-distribution [41]. Ligand environment modifications and /or presence of Ru(III/IV)-oxo species reflected different uptake pathways [42]. Finally, a more reducing environment in hypoxic tumor cells could affect the ligands nature around $\mathrm{Ru}$ atoms [43].

Respect to Ru compounds, gallium has a single biologically relevant (III) oxidation state and offers an easier case study for ligand exchange investigations. Hummer and coworkers exploited XAS and micro-XAS to probe a possible interaction with serum proteins such as human serum albumin, and apotransferrin, of a promising antitumoral Ga complex and its relative coordination environment [44]. EXAFS spectra at Ga K-edge for the metal complex in different buffer conditions were almost identical. The edge energy position and shape reported from XANES spectra in tissues are consistent with the absence of Ga ligands replacement [44]. Interestingly, Hummer's paper elegantly proved the absence of beam-induced damage associated to the high synchrotron photons flux comparing spectra acquired at low $\left(10^{10} \mathrm{ph} / \mathrm{s}\right)$ and high $\left(10^{12} \mathrm{ph} / \mathrm{s}\right)$ photon fluxes. Such strategy was also adopted by Levina and 
coworkers [45]. Finally, a strong correlation emerged from micro-SXRF analysis between Ga, Fe and $\mathrm{Zn}$ bio-distributions. While Fe and Ga correlation can be expected due their chemical similarity, $\mathrm{Zn}$ distribution results remains a future investigation point.

$\mathrm{Mn}$ (II) organic complexes are also very interesting candidates for XAS speciation studies. In a recent paper by Weekley and coworkers, micro-SXRF and XAS were applied to study small-molecule mimics of manganese superoxide dismutase (MnSOD) with potential therapeutic application in conditions linked to oxidative stress [46]. Using micro-SXRF it was possible to quantify a $10-20$ fold increase in $\mathrm{Mn}$ intracellular concentration after MnSOD mimics treatment, evidencing a correlation between increased complexes lipophilicity and uptake. A fine set of standards compounds was used to perform LCF of XANES region and EXAFS analysis on Mn complexes in cell media and in vitro. Remarkably, the thermodynamic stability of many $\mathrm{Mn}$ compounds tested did not reflect the experimental degree of biotransformation measured. Indeed, two important points could be highlighted. First, MnSOD mimics were found in cells in the intact form up to $85 \%$, i.e., as $\mathrm{Mn}$ (II) bound to the original organic ligands. The residue was metabolized over time into $\mathrm{Mn}$ (II) phosphate, modeled by a solid amorphous $\mathrm{Mn}$ (II) phosphate. Secondly, the generation of a stable intracellular $\mathrm{Mn}$ (III) species via the oxidation of an exogenously administrated $\mathrm{Mn}$ (II) compound was evidenced for the first time.

The speciation of vanadium-based pharmacological compounds have been studied in detail by Levina and collaborators. The authors carefully built one of the most accurate and comprehensive XAS database about vanadium compounds [45, 47]. Based on such a wide set of standards, several studies on the biotransformation of V-based antidiabetic $[45,47]$ and anticancer [48] compounds were performed. The presence of pre-edge features in V XAS spectrum simplifies the speciation analysis. The pre-edge peak is due to electronic transitions (mainly dipole allowed) to empty bound states near the Fermi level, and is sensitive to $V$ atom coordination number and oxidation state. As a consequence, Levina and his group measured 23 different standard samples reporting the resulting correlations of pre-edge height normalized, white line height normalized and energy pre-edge position on 3D graph $[45,47]$. Thanks to this effort, many consistent results were obtained by the XANES study of $V$ antidiabetic compounds under conditions that mimic oral administration. Indeed, typical anti-diabetic $\mathrm{V}(\mathrm{V})$ and $\mathrm{V}(\mathrm{IV})$ complexes underwent profound chemical changes in gastrointestinal media, including dissociation of the ligands, $V(I V)$ oxidation to $V(V)$ (in the absence of food), or $V(V)$ reduction to $V(I V)$ and even V(III) (in the presence of food) [47]. Important $V$ drugs biotransformation has been assessed also in red blood cells : uptake of $V$ compounds, either by active transport $\left(\left[\mathrm{H}_{2} \mathrm{VO}_{4}\right]^{-}\right)$or by passive diffusion ( $\mathrm{V}(\mathrm{IV})$ or $\mathrm{V}(\mathrm{V})$ complexes with organic ligands), and their conversion into well-defined $\mathrm{V}(\mathrm{IV})$ and $\mathrm{V}(\mathrm{V})$ metabolites; the presence of a more reducing environment for $\mathrm{V}$ than in isolated blood plasma [48]. In the most recent paper by Levina and collaborators, by means of XANES data analysis, a major roles was proposed for $\mathrm{V}(\mathrm{V})$ species, including $\mathrm{V}(\mathrm{V})$ peroxido complexes, as reactive intermediates responsible for both antidiabetic and cytotoxic activity of $\mathrm{V}(\mathrm{V})$ and (IV) complexes. Moreover, XANES spectroscopic studies revealed for the first time the capacity of mammalian cells to generate and maintain $\mathrm{V}(\mathrm{V})$ when treated with $\mathrm{V}(\mathrm{IV})$ complexes [48].

\subsection{Speciation of toxic elements}

The study of inorganic compounds effects on human health is one of oldest branch of applied toxicology. Modern orthopedics offers interesting XAS case studies [49-51]. Cobalt, chromium and relative alloys are the typical constituents of hips prosthesis but also the main responsible for hips failure and induced toxicity. In the paper of Harts and collaborators, the impact of two different kind of hips implants have been investigated by means of XANES and micro-SXRF on human tissue ex vivo. The XRF measurements proved that one set of samples had a relative concentration of Co 10-times 
higher compared to the other. Noticeably, a possible correlation between the relative Co increase and hips failure rate was also observed. The LCF analysis of Co XANES spectra region demonstrated that the two most abundant species were metallic $\mathrm{Co}$ and $\mathrm{Co}(\mathrm{II})$ as protein complex. Despite the sample number limitation, which is a common restraint in human studies, the authors finally correlated the amount of Co rather than its speciation as reason for the higher failure rate of one of the two set of hips implants [49]. Based also on Hart's paper, Shah and collaborators focused their micro-XAS and $\mathrm{XRF}$ investigations on a more practical in vitro system, testing both $\mathrm{Co}$ and $\mathrm{Cr}$ compounds metabolism in osteoblast and osteoclast cell lines. Many results were consistent with Hart's findings. First, both osteoblasts and mature osteoclasts showed the presence of $\mathrm{Co}$ in the $\mathrm{Co}$ (II) oxidation state, corresponding to the $\mathrm{Co}(\mathrm{II})$ acetate spectra, indicating that no potentially harmful intracellular redox reactions took place following $\mathrm{Co}^{2+}$ ions uptake in cells. Second, the similarity of $\mathrm{Cr}$ K-edge spectrum with $\mathrm{Cr}$ (III)OH and $\mathrm{Cr}(\mathrm{III}) \mathrm{PO}_{4}$ in both the cells lines confirmed the $\mathrm{Cr}$ (III) specie presence. Interestingly, more insights on $\mathrm{Cr} / \mathrm{Co}$ toxicity and uptake mechanism details were also provided. Indeed, micro-SXRF maps showed a $\mathrm{Co}$ (II) localization in the osteoclasts basolateral membrane suggestive of endocytosis process. Furthermore, cells treated with $\mathrm{Cr}$ (III) compound displayed a perinuclear metal distribution despite the fact that the absence of $\mathrm{Cr}$ (III) transporter in human cells for its uptake is considered as a dogma in toxicology. Such unexpected presence was explained by the authors as consequence of albumin mediated endocytosis. Another more expected result was obtained after cells treatment with $\mathrm{Cr}(\mathrm{VI})$ compounds. Indeed, $\mathrm{Cr}(\mathrm{VI}) \mathrm{K}$-Edge spectrum exhibits a typical pre-edge feature in the XANES region. The absence of such feature and the strict resemblance with $\mathrm{Cr}$ (III) spectrum were consistent with the intracellular bio-reduction model of $\mathrm{Cr}(\mathrm{VI})$ [51].

In the already cited orthopedics context, the dental restoration is indeed another field of XAS applications. In a Sugiyama and collaborators study, a combination of micro-SXRF, micro-PIXE (Particle Induced X-ray Emission), and XANES analyses was used in order to detect and identify trace metal elements contained in oral mucosa of oral cavity lesions [50]. This paper is based on the analysis of only two tissue sections from two different patients. As a consequence it can be considered as a "proof of concept" more than a comprehensive toxicological study. The paper reported the presence of many trace metals close to the inflammatory site, previously identified by means of histopathology labeling. $\mathrm{Ni}$ XANES spectrum resembled that of $\mathrm{Ni}(\mathrm{OH})_{2}$, while $\mathrm{Zn}$ and $\mathrm{Cu}$ were similar to metallic foil spectra suggestive of brass alloy presence.

Despite it is probably the most important toxicological case of the last 50 years, asbestos and its carcinogenic mechanisms are still poorly understood. Recently, Pascolo and coworkers using microSXRF imaging and micro-spectroscopic combination investigated the asbestos bio-persistent fiber aggregates (asbestos body) ability to impair Fe homeostasis in tissues [52]. The overall study was based on 10 different patients, with various grade of asbestos related pathologies, for a total of 40 tissue sections and 30 asbestos body analyzed. Noteworthy, the unique ability of micro-SXRF multiple element mapping allowed for asbestos body and shape determination from $\mathrm{Si}$ and Fe distributions respectively. Furthermore, $\mathrm{P}$ and $\mathrm{S}$ signals were successfully used to recognize cell morphologies in unstained histological sections. Pascolo's group clearly identified the presence of micro- and nanovesicles with relatively high Fe content in the proximity of the asbestos body and fibers-phagocyting alveolar macrophages. The relative Fe content in the body, vesicles and the near-by regions was up to 1000 times higher than the Fe content in non-affected tissue, proving a consistent unbalance in Fe metabolism. Moreover, XRF maps revealed a high amount of $\mathrm{P}, \mathrm{Ca}$ and $\mathrm{Mg}$ in the fiber casing, which suggested a correlation between calcification and Fe deposition. However, calcification seemed to be a consequence of phagocytosis due to low Ca presence in thin coated fiber regions. The inner nature of asbestos body was then revealed by means of micro-XAS analysis. The Fe K-edge spectrum LCF analysis confirmed that most of the iron detected around asbestos fiber (coating and ferruginous bodies) is compatible with the presence of ferritin, a typical Fe storage protein, and as +3 main 
oxidation state. Different asbestos bodies in different samples displayed an average composition of $71 \%$ ferritin (ferrihydrite), $16 \%$ haematite, $12 \%$ crocidolite, one of the mineral structure of asbestos fibers, and $1 \%$ metallic iron. The authors finally explained such speciation as an alteration of iron chemistry during asbestos body permanence [52].

In vitro study on Mn toxicity and related speciation was conducted by Carmona and collaborators [53]. Mn exposure alters the dopaminergic system which plays a critical role in the dysregulation of motor and cognitive functions, leading to a pathological condition known as manganism, a disease with symptoms resembling those of Parkinson's disease. The study tested by means of micro-XANES and micro-SXRF techniques different Mn compounds, with inorganic and organic forms, and with oxidation number ranging from (I) to (III). The compounds were representative of the most important environmental $\mathrm{Mn}$ sources, an organic fungicide, a combustion additive and its related combustion products. A preliminary cytotoxicity assessment was performed in the PC12 dopamine cell line to study correlation between toxicity and Mn speciation. For the micro-XANES investigation, a good set of standards could be measured evidencing differences in the edge position energy and shapes among different $\mathrm{Mn}$ oxidation states. Interestingly, an optical microscopy fluorescence labeling evidenced a precise correlation between Golgi compartment fluorescence signal and Mn distribution (Figure 3). Strikingly, the bioaccumulation in the Golgi was mostly independent from the administered $\mathrm{Mn}$ form, indicating such organelles are crucial for the Mn cellular storage and detoxification. A remarkable micro-XANES speciation limit of $10^{-17} \mathrm{~g}$ for single cell measurements could be estimated. Under $\mathrm{Mn}$ (II) compounds treatment, no redox state alteration were found in cells meaning that the metal toxicity did not rely on oxidation to $\mathrm{Mn}(\mathrm{III})$ state. $\mathrm{Mn}(\mathrm{I})$ compounds, instead, clearly underwent to oxidation in cell or cell media leading once again to the presence of $\mathrm{Mn}$ (II). After $\mathrm{Mn}$ (III) oxide administration, only $\mathrm{Mn}$ (III) could be found in cell cytoplasm resulting from untransformed solid $\mathrm{Mn}$ (III) particles uptake. $\mathrm{Mn}$ (III) oxide was the less toxic tested compound. This result could be due to the low solubility of the $\mathrm{Mn}$ (III) oxide specie. In the final picture, the authors showed that the Golgi apparatus plays a key role for $\mathrm{Mn}$ detoxification via exocytosis. From the comparison between cell viability assays and speciation analysis, moreover, the elevated toxicity of the fungicide compound seemed to be mainly related to its organic counterpart, rather to the metal moiety. Mn toxicity was directly related to solubility of the tested compounds. As for the previous case of Co toxicity, a clear indication of cellular bioaccumulation excess over speciation emerged as the reason for the Mn toxicity [53].
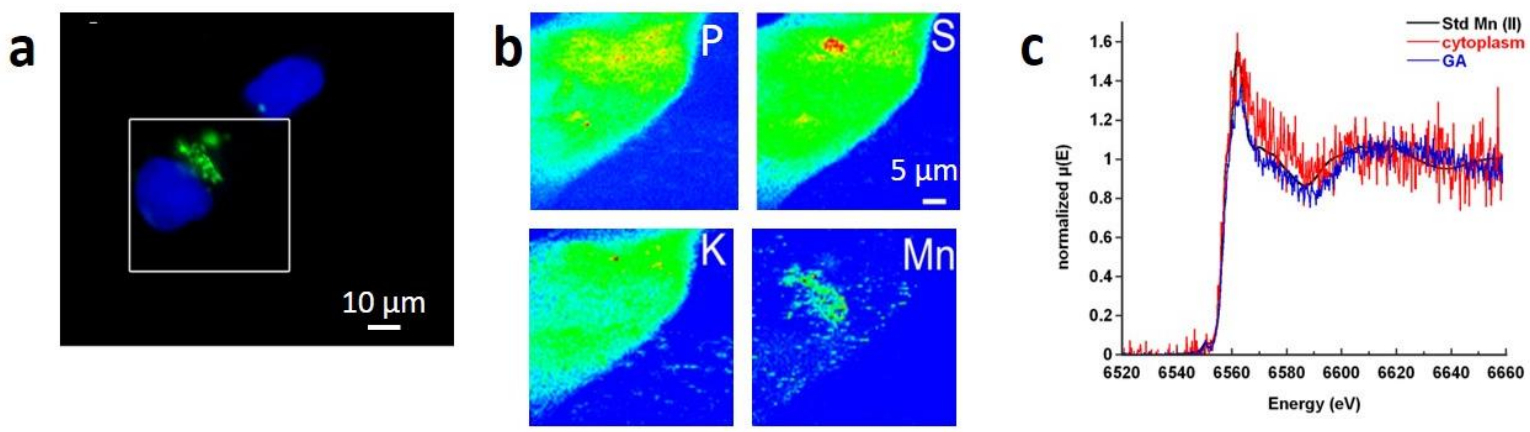

Fig 3. a) Optical fluorescence imaging of Golgi apparatus (GFP, green) and nucleus (Hoechst dye, blue) in living cells exposed to $500 \mu \mathrm{M} \mathrm{MnCl}_{2}$; b) SXRF element imaging of the cell identified in the white square, after cryofixation; c) Micro-XANES at Mn absorption K-edge at the organelle level, in Golgi apparatus (GA, blue line), cytoplasm (red line), and from a $\mathrm{Mn}(\mathrm{II})$ reference standard (black line), analysis performed at liquid nitrogen temperature in a frozen hydrated cell. Adapted from Ref. [53] with permission from The Royal Society of Chemistry. 
A remarkable and very recent example of toxicological study supported by XAS is offered by Pierrefite-Carle and collaborators [54]. The primary sites of uranyl ions $\left\{\mathrm{U}(\mathrm{VI}) \mathrm{O}_{2}\right\}^{2+}(\mathrm{U}(\mathrm{VI})$ for simplicity) accumulation are bones, where a permanent storage takes place potentially leading to a long term toxicity. As shown by the authors, $\mathrm{U}(\mathrm{VI})$ treatment reduced the calcification reaction by means of an autophagy process blockage. TEM measurement confirmed the $U$ accumulation in needle-like structures inside autophagic vesicles. In this context, EXAFS spectroscopy performed on cell media and cells pellets provided fundamental details to understand a possible competition between $\mathrm{Ca}$ and $\mathrm{U}(\mathrm{VI})$ in the biomineralization process. The cell media $\mathrm{k}^{2}$-weighted EXAFS spectrum, in agreement with the theoretical thermodynamic simulation data, was consistently modeled with a carbonate environment. Besides oxygen coordination at first and second shells, 1.5(5) carbon has also been found in the third coordination sphere, suggesting that about two carbonate anions were linked to $\mathrm{U}$ in a bidentate mode. However, a Ca atom addition to the model (at $4.1 \AA$ ) did not improve significantly the quality of the fit. As a consequence, it was not possible to further refine the speciation and discriminate between three forms of carbonate theoretically predicted. EXAFS $k^{2}$-weighted spectrum cells evidenced clear distinctions respect to the cell medium, in particular in the third coordination shell region. Indeed, a simple carbonate ligation model could not appropriately fit the experimental data but a meta-autunite model $\mathrm{Ca}\left[\left(\mathrm{UO}_{2}\right)\left(\mathrm{PO}_{4}\right)\right]_{2}\left(\mathrm{H}_{2} \mathrm{O}\right)_{11}$ could provide a satisfactory fit. The combination of two sets of EXAFS data proved the biotransformation of uranium carbonate species into a meta-autunite phase upon uptake by osteoblast cells. In conclusion for this study, the EXAFS measurement strongly supported the authors model for $\mathrm{U}$ mediated toxicity. Indeed, $\mathrm{U}(\mathrm{VI})$ competition with Ca was consistent with the calcification process reduction and leaded to the accumulation of meta-autunite crystals responsible of autophagy block. Such block with potential increase of intracellular ROS is indicated as the final source of $U$ toxicity.

\subsection{Speciation of metallic nanoparticles}

Metallic nanoparticles (NPs) huge impact in the biomedical context has fostered the exploitation of synchrotron techniques in this field. An exhaustive review of the vast literature is out of the scope of the present review. Two excellent recent articles provide a precise glimpse of the currents trends [55-56]. Despite that, a shift in the scientific attention has to be pointed out, from the initial $\mathrm{Au}$ and Ti oxide NPs biotransformation study a variety of recent papers is now focusing on silver NPs. As a consequence, we will revise mainly the very recent findings which are not present in the prior reviews with particular consideration of Ag NPs speciation.

Nevertheless, despite already reported in the cited reviews, we will begin our commentary from Wang's work on gold NPs. Such paper represents an excellent example of XAS applied to nanomaterial investigations due to its clarity and accuracy. Indeed, the combination between XAS and conventional techniques such as TEM or molecular simulation contributed to highlight many aspects of NPs-cells interactions. Au NPs show an extreme versatility in biomedical context: radiotherapy, biosensors, drug delivery are the most influenced fields. As a consequence, in vitro investigations of NPs-cellular proteome interaction is of primary importance. Protein corona consists in groups of peptides adhering on nanomaterial surface and able to mediate the nanostructure uptake inside the cells. Wang and collaborators applied XAS and SXRF techniques in order to study binding structure and stability of Bovine Serum Albumin (BSA) corona on gold nanorods (AuNRs) [57]. S K-edge and Au L III-edge XANES were exploited in order to assess the possible BSA adsorption mode on Au surface. Remarkably, as deeply addressed in the next paragraphs, XANES data analysis is a fully reliable method for sulfur 
speciation study in both nanomaterial or physiopathology studies. As reported in a summary picture in Figure 4, the XANES LCF data analysis was performed on BSA-AuNRs spectrum using a combination of proper standard set including the three possible sulfur species in the system: thiol (R-S-H, R-S-R thiol), disulfide (S-S), and Au-thiol coordination ( $\mathrm{Au}-\mathrm{S}$ ). The analysis showed that the BSA corona adsorption on AuNRs decreased the content of disulfides from $82 \%$ to $58 \%$ by inducing $26 \%$ of the sulfur atoms to transform into Au-S. Such changes in BSA sulfur species suggested that the BSA-AuNRs interaction included initial electrostatic adsorption followed by $\mathrm{Au}-\mathrm{S}$ coordination with a consequent secondary structure alteration. Respect to $S$, Au L(III)-edge XANES did not show any changes in the chemical form of AuNRs gold due to very low number of coordinated atoms respect to total gold atoms [57].
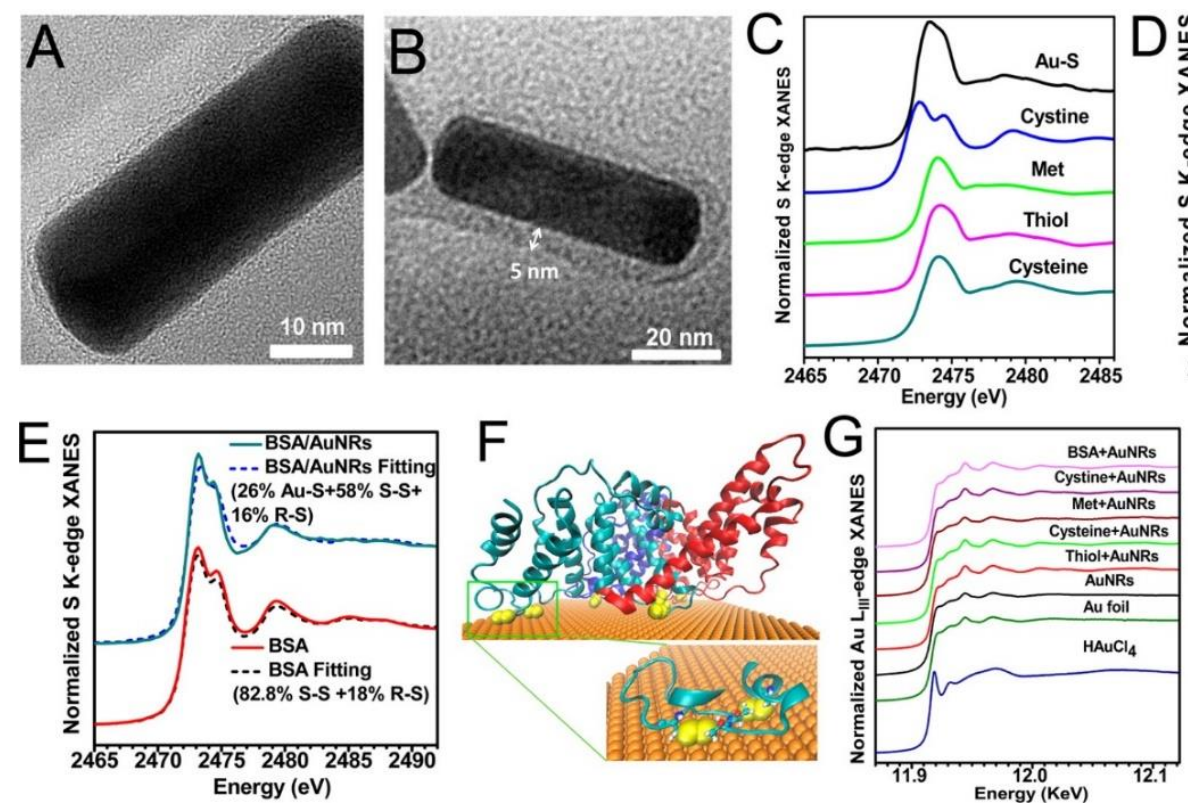

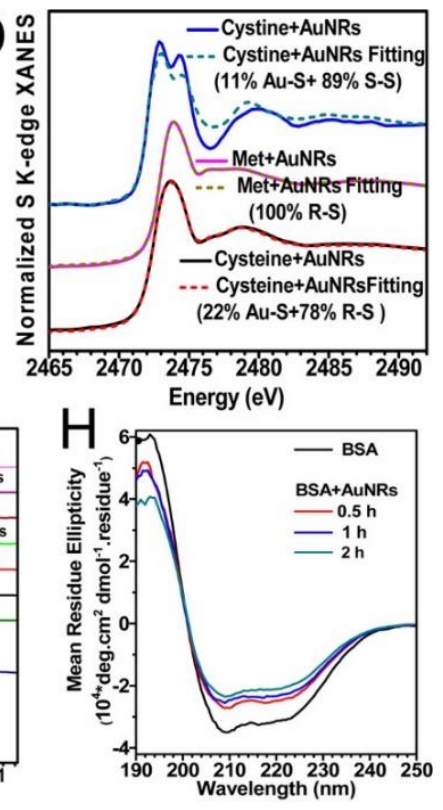

Fig. 4. (A) Characterization of AuNRs and (B) BSA corona-coated AuNRs according to TEM. (C) Sulfur species used as standard: Au-S, R-S (cysteine, thiol, Met), and R-S-S-R' (cystine), shown as normalized $S$ K-edge XANES spectra. (D) Chemical species of sulfur in cysteine, Met, and cystine after incubation with AuNRs. (E) Composite of sulfur chemical species in BSA and the BSA corona on AuNRs. (F) Molecular simulation showing disulfides of BSA (yellow) binding to the Au (111) surface of AuNRs. (G) Normalized gold L-(III) edge XANES of reference samples (Au foil and $\mathrm{HAuCl}_{4}$ ) and other samples including AuNRs and AuNRs incubated with BSA, cysteine, cystine, and thiol, respectively. (H) Circular dichroism spectra of BSA bound to AuNRs. Adapted and reprinted with permission from [57]. Copyright 2013 American Chemical Society.

Recently, Ag NPs are taking the spotlight due to their promising antibacterial activity. However, employed as doping material or as drug carrier, AgNPs have risen important human toxicity issues. Interestingly, as for Au case Wang and coworkers published a detailed paper relying on XAS techniques for Ag NPs speciation study on monocytes [58]. Indeed, due to their blood clearance activity, monocytes and macrophages are among the most exposed cells types to an AgNPs in vivo treatment and used as cellular model by many groups. Davidson and coworkers investigated Ag NPs speciation in rat lung by means XAS [59]. In their article, an accurate EXAFS study was performed on lung tissues from rats following a 6-h inhalation exposure to aerosolized 20 and $110 \mathrm{~nm}$ diameter AgNPs. The samples were also representative of different time of NPs lung presence (up to 7 days after the 
treatment). Interestingly, all the samples showed one dominant peak between 1 and $3 \AA$ in the real space, corresponding to the first $\mathrm{Ag}-\mathrm{Ag}$ coordination shell. On the basis of such information, the authors concluded that Ag species in all the samples were dominated by pristine metallic Ag. Despite that, the first shell fit proved a progressive reduction over the time of the coordination number $(\mathrm{N})$ from the $\mathrm{N}=12$ (theoretical crystalline Ag bulk), to a 3-5 range. Such phenomenon, qualitatively correlated to the first peak amplitude reduction, was more evident for the smaller particle and considered by the authors as indicative of NPs modification [59]. As mentioned before, Wang and coauthors replicated the in vitro study for Au with AgNPs system obtaining once again very exhaustive results. In Wang's paper, Ag K-edge XANES LCF analysis results were reported as a function of AgNPs uptake/exocytosis time. A clear elemental distribution alteration during the NPs biological processing was evident. Indeed, original AgNPs contained 93.1\% elemental $\mathrm{Ag}(0)$ and $6.9 \% \mathrm{Ag}-\mathrm{O}$. During internalization and exocytosis a progressive substitution of $\mathrm{Ag}-\mathrm{O}$ with $\mathrm{Ag}-\mathrm{S}$ was reported leading to final $21.4 \%$ of $\mathrm{Ag}(0)$ against $78.6 \%$ of Ag-S. The transient comparison of $\mathrm{Ag}(\mathrm{I})$ specie was also described. Finally, XANES data in combination with TEM and 3D tomography imaging allowed for the identification of a possible AgNPs toxicity mechanism. Indeed, after the internalization, AgNPs underwent to a progressive dissolution to $\mathrm{Ag}^{+}$ions. Therefore, the consequent ions dispersion and $\mathrm{S}$ binding could lead to cytotoxic ROS production or apoptosis pathway triggering. In addition, the NPs multistep dissolution process, due to its relatively slow rate, could also explain the lower cytotoxicity of AgNPs compared to $\mathrm{Ag}^{+}$ions administration [58]. Similar results were obtained also by Jiang and collaborators on Chinese hamster ovary cells [60]. A further confirmation of macrophage role in AgNPs clearance and toxicity was made by Smulders and collaborators [61]. Compared to other works, the authors provided the Ag lung biodistribution and speciation in vivo of different size NPs by means of XRF, XANES, and PIXE techniques combined approach. Moreover, the local concentration, and the colocalization with other elements such as $\mathrm{Fe}, \mathrm{Cu}$ and $\mathrm{S}$ was also reported. $\mathrm{L}$ (III)-edge XANES spectra were acquired on three Ag-rich spots identified by XRF and Ag speciation was determined by LCF. In two spots, $\mathrm{Ag}$ was still partially present as elemental $\mathrm{Ag}(0) 37 \%$ and $\mathrm{Ag}^{+} 55 \%$ respectively, while the other spot showed $100 \% \mathrm{Ag}^{+}$ions complexation to thiol-containing molecules. Significant higher concentrations of $\mathrm{Cu}, \mathrm{Fe}$ and $\mathrm{S}$ were found in the $\mathrm{Ag}$-rich spots. The $\mathrm{Cu} / \mathrm{Ag}, \mathrm{Fe} / \mathrm{Ag}$ and $\mathrm{S} / \mathrm{Ag}$ ratios were much higher than in the original NPs confirming that such elements did not come from sample impurities. The most important data however, came from SXRF analysis and histology comparison: first, a local macrophage increase in the lungs was observed. Second, Ag NPs presence was shown in approximately a quarter of all macrophages in the lumen [61].

As previously mentioned, the $\mathrm{Ag}(0)$ to $\mathrm{Ag}^{+}$dissolution and the consequent complexation with biological thiols is the main key to unravel Ag toxicity. Veronesi and coworkers in series of very interesting articles deeply investigated the chemical nature of Ag-S bond and its relationship with cellular environment [62-64]. 

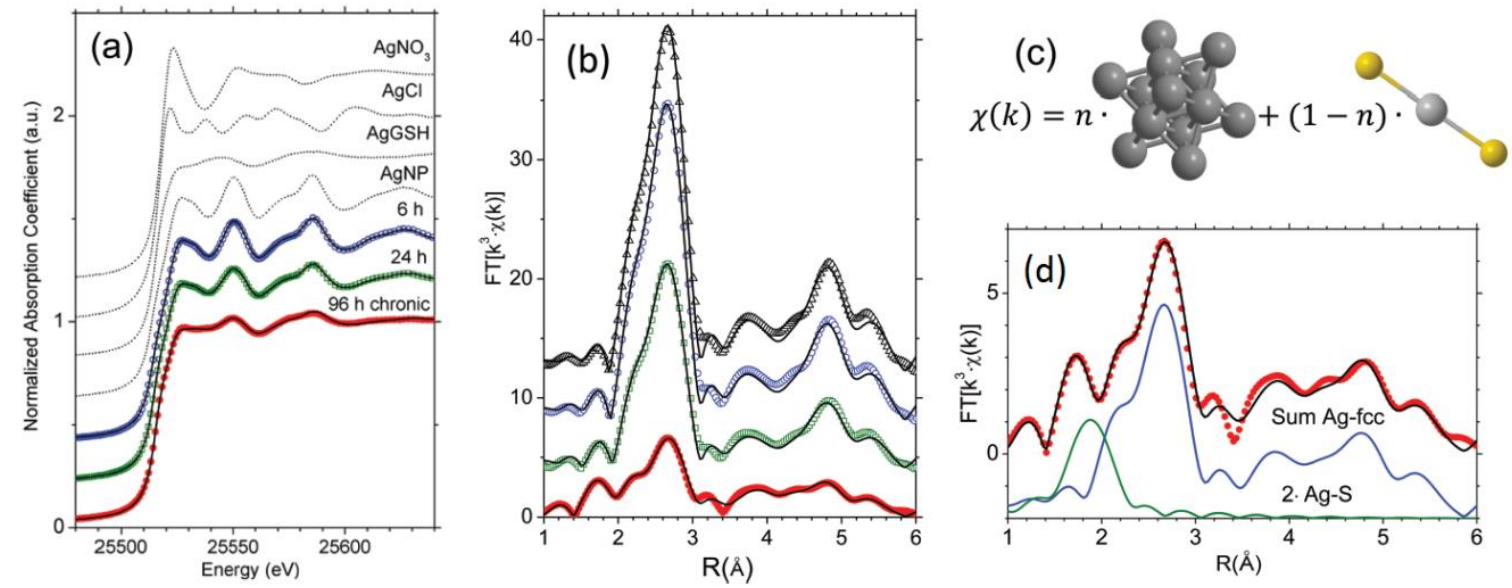

Fig. 5. (a) Experimental Ag K-edge XANES spectra of the reference compounds and samples. Fouriertransformed experimental Ag K-edge EXAFS spectra of silver nanoparticles and of primary murine macrophages exposed to silver nanoparticles. (b ) FT $k^{3}$ weight EXAFS spectra of NPs at different time and relative fits (c) Pictorial representation of the two components ab initio model used for fitting the data: grey atoms $=\mathrm{Ag}$, yellow atoms $=\mathrm{S}$. (d) Example of Relative importance of the Ag-fcc (solid blue line) and $\mathrm{AgS}_{2}$ (solid green line) components on fit (solid black line). Adapted from [62] with permission from The Royal Society of Chemistry.

As reported in Figure 5, Ag K-Edge XANES region comparison evidenced a clear qualitative trend consisting in the progressive dumping of the signal as a function of time. Such alteration was evocative of AgNPs modification chemistry. LCF analysis provided a quantitative explanation of the spectra alteration suggesting a progressive formation of Ag-glutathione (AgGSH) soluble species. Interestingly, the authors pointed out that to the intracellular Ag dissolution did not correspond to an increase in cell mortality, and on the contrary, $\mathrm{Ag}$-thiol complexes formation as potential $\mathrm{Ag}^{+}$scavenger was envisaged. In order to finally prove the formation of AgGSH intracellular species and to determine its relative amount, a brilliant analysis of the EXAFS signal was performed. Briefly, models were built on $a b$ initio simulations for $\mathrm{Ag}$ in the face centered cubic crystal structure (Ag-fcc) and $\mathrm{Ag}$ recombined with organic S (Ag-rec). Such models were then refined on the AgNP and AgGSH standard spectrum, respectively, and used to fit the experimental data in the way that their weights sum to one according to the formula below (Eq. 6):

$$
\chi(k)_{\mathrm{Ag}-\exp }=n \chi(k)_{\mathrm{Ag}-\mathrm{fcc}}+(1-n) \chi(k)_{\mathrm{Ag}-\mathrm{rec}}
$$

where $\chi(k)_{A g-e x p}$ is the experimental EXAFS function of Ag in cellular samples, $\chi(k)_{A g-f c c}$ and $\chi(k)_{A g-r e c}$ are the theoretical EXAFS functions generated for the Ag-fcc and Ag-rec models respectively, and $\mathrm{n}$ is the fraction of $\mathrm{Ag}$ in undissolved NPs.

Noteworthy, EXAFS signal final results analysis were consistent with the XANES LCF ones. Moreover, $\mathrm{Ag}-\mathrm{S}$ distance in recombined $\mathrm{Ag}^{+}$in the $24 \mathrm{~h}$ and $96 \mathrm{~h}$ exposed samples showed values of $2.41 \pm 0.03 \AA$ and $2.38 \pm 0.01 \AA$ respectively, close to the $\mathrm{Ag}-\mathrm{S}$ distance of $2.400 \pm 0.005 \AA$ of $\mathrm{Ag}-\mathrm{GSH}$ complex and incompatible with the average value of $2.51 \pm 0.05 \AA$ typical of $\mathrm{AgS}_{3}$ coordination complex or Ag-metallothionein $(2.44 \pm 0.03 \AA$ ). As a consequence, the authors finally claimed glutathione as the most probable ligand for $\mathrm{Ag}^{+}$binding in macrophage cellular environments [62]. In a more recent paper by the same authors the above theoretical model was applied in order to study Ag NPs fate in hepatocytes. Interestingly, comparing to macrophage study, a bond length increase up to $2.44-2.45 \AA$ 
was reported for the AgS complex. Such increase was consistent of Ag-S trigonal sites, typical of metallothioneins $\mathrm{Ag}$ chelation [63], and suggestive of NPs fate dependence on a precise protein expression and metabolism. Moreover, dissolution process was faster for citrate-stabilized than for PolyVinilPyrrolidone (PVP)-coated NPs. Noticeably, nanometric resolution XRF images allowed for NPs aggregates visualization in cellulo. A uniform weak signal due to Ag was recorded throughout the whole cells after exposure for both citrate- and PVP-coated Ag NPs. This signal was comparable to the one due to ionic $\mathrm{Ag}$ and highlighted the $\mathrm{Ag}^{+}$concentration slow increase inside the cell upon $\mathrm{AgNPs}$ exposure. Moreover, such data was consistent with the expected lower toxicity of Ag NPs compared to $\mathrm{AgNO}_{3}$ [64].

The most recent insights on NPs dissolution dynamic process were provided by Roman and collaborators. The authors investigated AgNPs integrity during bloodstream systemic distribution on plasma samples after NPs treatment [65]. Selected on the basis of preliminary SXRF mapping, the Ag, $\mathrm{Cl}$ and $\mathrm{S}$ distribution was acquired in the spatial region surrounding NPs. A characteristic non-uniform $\mathrm{Cl}$ accumulation was observed at the $\mathrm{Ag}$ particles border, while $\mathrm{S}$ was uniformly distributed in the nearby medium. Finally, the LCF XANES data analysis on the integrated XRF region showed an elemental distribution of $53 \% \mathrm{AgCl}, 31 \% \mathrm{AgNPs}$ and $15 \%$ Ag-thiols. In authors' opinion, such unexpected $\mathrm{AgCl}$ phase presence could be explained by kinetics reasons. Indeed, although protein thiols should be thermodynamically favored for $\mathrm{Ag}$ binding as well described by the others authors cited during this review, $\mathrm{Cl}$ atoms could have a kinetic advantage when interacting with $\mathrm{Ag}$ ions directly on the charged surface of the bare NPs, leading to an early effect of partial dissolution. Despite the reliability of Roman's data, in order to confirm such hypothesis many other experimental conditions should be tested since as discussed before, $\mathrm{pH}$ but also proteins media composition can deeply affects NPs solubility [58,62-64].

Due to XAS multi element probe nature, a variety of different metallic nanomaterials, iron, copper, zinc and more recently iridium biotransformation have been successfully described. In particular, Tien and collaborators exploited the great sensitivity of Fe K-edge XANES and EXAFS to assess the $\mathrm{Fe}_{3} \mathrm{O}_{4} \mathrm{NPs}$ intracellular stability after $\mathrm{SiO}_{2}$ encapsulation in a mesenchymal cell line. Such work was set in the framework of $\mathrm{Fe}_{3} \mathrm{O}_{4}$ based NPs exploitation as the magnetic resonance imaging (MRI) contrast agent. Noteworthy, comparing TEM and XRF images further details about NPs distribution were unraveled. First, $\mathrm{Fe}_{3} \mathrm{O}_{4}$ and $\mathrm{Fe}_{3} \mathrm{O}_{4} @ \mathrm{SiO}_{2}$ NPs were shown to both locate predominantly in the cell cytoplasm. LCF XANES analysis on $\mathrm{Fe}_{3} \mathrm{O}_{4} \mathrm{NPs}$ treated cells showed that up to 14 days after the treatment, $79 \%$ of intracellular Fe was retained as $\mathrm{Fe}_{3} \mathrm{O}_{4}$ species and the rest as ferritin-like species. Further confirmation of elemental speciation came by the Fe-Fe peak decrease in the EXAFS Fourier-transformed $k^{3}$-chi( $(k)$ functions of $\mathrm{Fe}_{3} \mathrm{O}_{4} \mathrm{NPs}$. On the other hand, $\mathrm{Fe}_{3} \mathrm{O}_{4} @ \mathrm{SiO}_{2}$ showed a superb stability after a week with no apparent modification of the spectrum. Despite that, after 14 days a $10.8 \%$ of ferritin-like species was found. In both kind of NPs, the culture medium supernatants analysis revealed that most of the excreted NPs remained in their original $\mathrm{Fe}_{3} \mathrm{O}_{4}$ form while the rest were present as $\mathrm{Fe}_{2} \mathrm{O}_{3}$ species probably due to an extracellular $\mathrm{Fe}_{3} \mathrm{O}_{4}$ oxidation. Tien's data illustrated that $\mathrm{Fe}_{3} \mathrm{O}_{4} \mathrm{NPs}$ presented a remarkable stability in cells and that $\mathrm{SiO}_{2}$ clearly contribute to increase the NPs inertness [66]. Interestingly, a ferritin-like species presence after Fe based NPs exposure was also confirmed independently by Wang and collaborators in brain samples after intranasal instillation [67].

As already seen with $\mathrm{Ag}$ and Fe examples solubility and speciation are two determinant factors for the understanding of NPs toxicity. In this context, Ivask and collaborators have applied XANES spectroscopy to assess $\mathrm{ZnO}$ CuO NPs biotransformation in human T-lymphocyte cells and in their exposure medium over a $24 \mathrm{~h}$ time period [68]. This investigation was conceived as a proof of concept for extending the knowledge about NPs speciation and relative toxicity to other metals such as $\mathrm{Zn}$ and $\mathrm{Cu}$. The study showed that $\mathrm{Zn}$ (II)-cysteine-like complexes (the most thermodynamically favored) and 
Zn-phosphate were the prevailing Zn species ranging $30-60 \%$ and $31-50 \%$ respectively. A relatively low fraction of $\mathrm{ZnO}$ and $\mathrm{Zn}$-acetate were found in the error limit and could not be considered reliable while $\mathrm{Zn}$ in cell culture medium was mainly in the form of $\mathrm{Zn}$-phosphate and $\mathrm{Zn}$-citrate. Interestingly, the final results showed high degree in similarity between $\mathrm{ZnO}$ and soluble $\mathrm{Zn}$ cell treatment suggesting a NPs fast conversion in cell culture medium followed by cell mediated transformations. Also $\mathrm{Cu}$ speciation both in cells and medium, was affected by the total transformation of CuO particles. The prevailing species in $\mathrm{CuO}$ and soluble $\mathrm{Cu}$ exposed cells was $\mathrm{Cu}$-cysteine (55-58\%). However, a small fraction of $\mathrm{Cu}$-histidine (11-20\%), and $\mathrm{CuO}$ (15-22\%) was also reported [68].

Finally, despite the lack of consistent sample amount, preliminary data on iridium NPs speciation were reported by Buckley and collaborators [69]. In this work, elemental maps of lung rats exposed to different Ir NPs sizes were produced by means of laser ablation inductively coupled plasma mass spectrometry (LA-ICP-MS) and XRF. The XRF elemental maps of lung tissue and broncho alveolar lavage fluid (BALF) cells showed Ir presence inside cells, at short and long times post-exposure. XANES analysis of the most concentrated XRF iridium spots in a lung sample at 1 day post-exposure and a BALF sample at 598 days post-exposure showed almost negligible modifications in the chemical speciation, suggesting limited if any dissolution.

\subsection{Speciation of essential elements}

One of the important applications of XAS in Biology is the in situ investigation of the redox chemistry of sulfur [30,36,70-71], as also illustrated in sections 3.5. and 3.6. Sulfur in biological systems can cycle through a large variety of oxidation states, from $(-2)$ as in sulfide, $(-1)$ in disulfide, $(0)$ in elemental sulfur, $(+4)$ in sulfite, $(+5)$ in thiosulfate, and up to $(+6)$ in sulfate [72]. As illustrated in Figure 6 , XANES at sulfur K-edge is particularly suited to the identification of sulfur species thanks to the large energy range over which the K-edge of specific electronic states of sulfur absorb (14 eV), and owing to the clear energy shifts of the absorption edges of the different sulfur species of biological interest. Direct speciation analysis of $\mathrm{S}$ using K-edge XANES can therefore provide rich chemical information owing to the existence of distinct resonance peaks with energy positions in linear correlation with the oxidation state of the $\mathrm{S}$ species. Despite the complex redox chemistry of sulfur, peak deconvolution and redox species identification can be performed accurately by means of computational methods like Gaussian curve fitting or by comparison with standard compounds followed by $\operatorname{LCF}[30,36,73]$. 


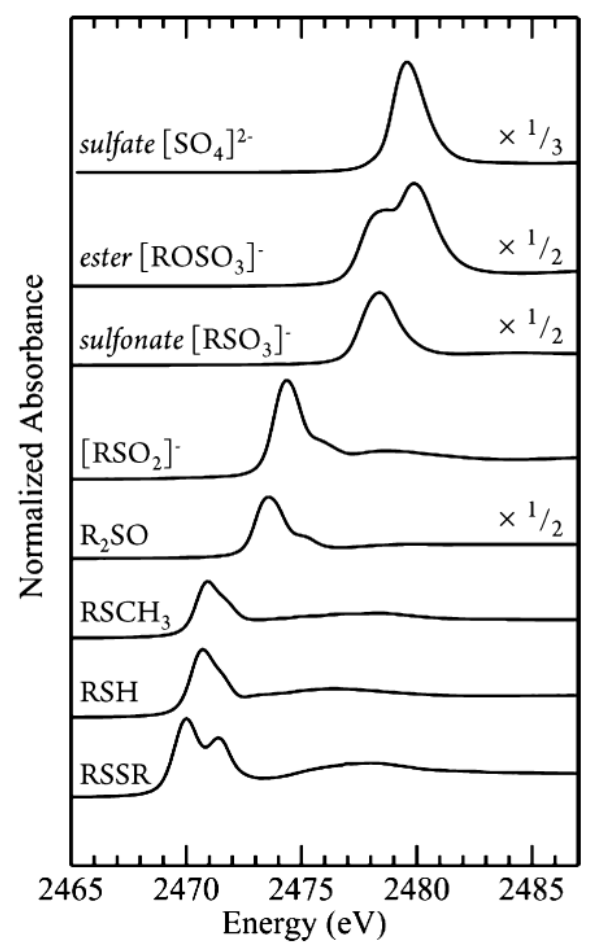

Fig. 6. Sulfur K-edge absorption spectra of representative sulfur species in aqueous solution. Reprinted with permission from [30]. Copyright 2012 American Chemical Society.

Sulfur speciation can inform about redox changes in biological tissues that may occur during pathological processes or following physiological modifications. For example the lattice-like arrangement of collagen fibrils required for corneal transparency is controlled, in part, by sulfated proteoglycans during embryogenesis. The study of corneal development during embryogenesis in chicks was performed using the ID21 X-ray microscopy beamline at the ESRF showing developmentally regulated alterations in thiols, organic monosulfides, ester sulfate, and inorganic sulfate species [74]. A detailed S speciation analysis using Gaussian curve fitting of XANES spectra is presented in a further study of corneal tissue from adult bovine from the same authors [36]. Four main peaks were distinguishable in the XANES spectra (Figure 7). This study illustrates the unique capability of S K-edge XANES to unveil complex $\mathrm{S}$ redox biological chemistry. Their energy positions indicate the chemical forms of $S$ with respectively organic monosulfides (R-S-R) and thiols (R-S-H) at $\approx 2,473.6 \mathrm{eV}$; sulfoxide groups $\mathrm{R}-\mathrm{S}(=\mathrm{O})-\mathrm{R}$ at $\approx 2,476.3 \mathrm{eV}$; sulfonate $\mathrm{R}-\mathrm{S}(=\mathrm{O})_{2} \mathrm{O}^{-}$at $\approx 2,481.1 \mathrm{eV}$; and sulfates $\mathrm{SO}_{4}{ }^{2-}$ at $\approx 2,482.7$ $\mathrm{eV}$. The feature at $\approx 2,497.0 \mathrm{eV}$ arises from cumulative multiple scattering contributions of all $\mathrm{S}$ species. A Gaussian curve fitting approach was undertaken for quantitative analysis resulting in $41 \pm 1 \%$ contribution from thiols and organic monosulfides, $8 \pm 1 \%$ from sulfoxides, $9 \pm 1 \%$ from sulfonates, and $42 \pm 1 \%$ from sulfates; some differences in corneal regions were observed. 


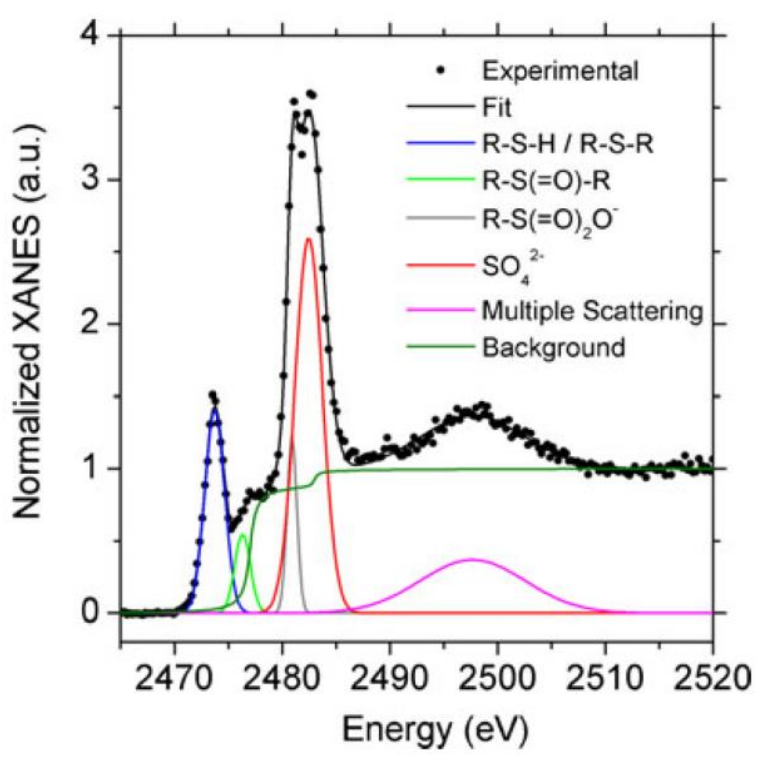

Fig. 7. Sulfur speciation analysis in a biological tissue. Example of fit (continuous black line) to the experimental data (black dots) acquired at S K-edge energy from a bovine corneal tissue sample. The individual curves composing the fitting model are reported as colored lines. Adapted from [36] with permission from Springer.

Another useful application of XANES is the speciation of trace elements in human calcifications. For example, strontium can substitute for calcium in bio-mineralization processes and strontium could thus either be involved in pathological conditions such as the initiation of calcium stone formation, or could help to prevent bone osteoporosis. Speciation of strontium can be obtained by fitting XANES spectra from unknown samples against spectra of known samples. Using this data treatment method, XANES analysis of human kidney stones showed that $80 \%$ of the strontium was present as strontium apatite and $20 \%$ as strontium carbonate, as performed at the Advanced Light Source [75]. XAS investigation of strontium coordination in bio-calcifications can provide further details about local structural organization and Sr potential implication in pathological progression. For example, XANES at Sr K-edge has been used at SOLEIL synchrotron to investigate whether Sr ions are simply adsorbed at the surface or inserted within apatite from pathological and physiological calcifications [76]. The results of this study concluded that there was no clear difference between XANES spectra from pathological and physiological samples. Zinc is another element of interest in the field of bio-mineral calcifications that could be involved in bone turnover. Micro-XANES at Zn K-edge was performed at SOLEIL synchrotron on meniscus from patients undergoing total knee joint replacement for primary osteoarthritis [77]. The results showed that at least two $\mathrm{Zn}$ species were present, one embedded in proteins, and a second one that could be associated to a $\mathrm{Zn}$ trap at the surface of the calcification. This conclusion was simply deduced from the comparison of $\mathrm{Zn}$ K-edge XANES spectra with those from standards compounds, $\mathrm{Zn}$ embedded within metalloproteins, and $\mathrm{Zn}$ in kidney stones where $\mathrm{Zn}$ is known to be located at the surface of the apatite.

An original study on bromine speciation in biological fluids and tissues was carried out at the Australian synchrotron at $\mathrm{Br}$ absorption K-edge [78]. In this study XANES was performed to investigate potential $\mathrm{Br}$ biotransformation. Although $\mathrm{Br}$ is not considered as an essential element it may participate to biological responses and it is known to exchange with chlorine and iodine ions in tissues. The potential demonstration using XANES that $\mathrm{Br}$ could exist in different chemical species in biological tissues would indicate that its presence is more than simply adventitious. Analysis of XANES spectra from bovine ovary tissues led to the conclusion that the predominant form of bromine was the $\mathrm{Br}^{-}$ion, 
as shown by the similarity of XANES spectra with the $\mathrm{NaBr}$. Since no biotransformation of bromine was evidenced, this study suggested that a biological role for $\mathrm{Br}$ was not apparent.

The possibility to perform element speciation on samples from human patients is obviously an important achievement for the understanding of the molecular mechanisms that could be altered in diseases related to elemental dyshomeostasis. This is for example the case for a recent report on $\mathrm{Cu}$ speciation of a liver biopsy from a Wilson's disease patient [79]. Wilson's disease is a genetic disorder due to a mutation in $A T P 7 B$ gene which codes for an ATPase that transports copper. The mutation causes the accumulation of copper in cells resulting in severe liver and brain dysfunction. In the study from Hachmöller et al., Cu distribution using micro-XRF and Cu speciation using XANEs were combined. XANES measurements were carried out at BESSY II synchrotron, at $\mathrm{Cu}$ K-edge on a liver biopsy from a Wilson's disease patient, and on two control samples. $\mathrm{Cu}(\mathrm{I}) / \mathrm{Cu}(\mathrm{II})$ ratios were determined by estimating the maxima of the first derivative of the XANES spectra and by comparison with those of $\mathrm{Cu}(\mathrm{I})$ and $\mathrm{CU}(\mathrm{II})$ standard compounds. In the liver biopsy from Wilson's disease patient a mixture of $\mathrm{Cu}(\mathrm{I})$ and $\mathrm{Cu}(\mathrm{II})$ was identified. However, the concentration of $\mathrm{Cu}$ was too low in control samples to determine the $\mathrm{Cu}$ oxidation state. This study gives some important indication on $\mathrm{Cu}$ oxidation state in Wilson's disease and at the same time underline the difficulty to perform XANES on Cu in biological samples due to its low concentration.

\subsection{Speciation of essential elements in carcinogenesis}

As discussed in section 3.1, XAS has numerous applications in anticancer drug development. It is also a tool to investigate the mechanisms of action of carcinogenic elements (section 3.2). In the field of cancer research, another domain where XAS can provide useful information is the speciation of essential elements as markers of tumor progression, or of elements involved in the natural prevention of carcinogenesis. Sulfur is one of the elements which speciation is suspected to be altered during carcinogenesis. A micro-XANES study at sulfur K-edge of human glioma tissue sections pointed out that in all the examined tissue sites, within cancer cells and outside cancer cells, sulfur is present in the (-2) oxidation state. The evaluation based on the white line position method showed that the studied spectra were similar to those of cysteine, methionine, and reduced glutathione [80]. This study settled down the analytical basis for sulfur speciation in neoplastic lesions but however did not compare the obtained data with those of control healthy tissue. Recently, Kumar et al. [81] investigated sulfur speciation in human cultured cells comparing normal fibroblast cells co-cultured, or not, with breast cancer cells. Cancer stimulated fibroblasts may play a role in the progression of tumors by enhancing the secretion of growth factors. The quantitative determination of sulfur chemical species from XANES spectra was carried out by fitting a linear combination of model sulfur compounds. The relative concentrations of thiol, thioether, disulfide, sulfoxide, sulfinic acid, sulfonic acid, O-linked sulfate ester, $\mathrm{N}$-linked sulfate ester, and inorganic sulfate were obtained. A significant increase in the amounts of thiols and thio-ethers was observed in the normal fibroblasts co-cultured with the cancer cells which is consistent with the higher cellular activity and proliferation. In this study calcium K-edge spectroscopy was also carried out on bulk samples using $\mathrm{Ca}_{2} \mathrm{P}_{2} \mathrm{O}_{7}, \mathrm{CaCO}_{3}$, hydroxyapatite, $\mathrm{Ca}(\mathrm{OH})_{2}$ and $\mathrm{CaO}$ as model compounds for relative quantification of calcium species. The authors observed that the calcium pyrophosphate, hydroxyapatite and calcium hydroxide concentrations increased in the cancer stimulated fibroblasts together with a decrease of relative calcium carbonate concentrations. This result might be related to the formation of micro-calcification in breast cancer where calcifications are composed of calcium phosphate containing pyrophosphate and hydroxyapatite.

Although present at much lower concentrations in the human body $\left(<1 \mu \mathrm{g} \cdot \mathrm{g}^{-1}\right)$, selenium is another chalcogen which displays a rich redox speciation in human cells. It is an essential trace element 
known to possess cancer chemo-preventative properties, and is present in a wide variety of metabolites in cells, with organic and inorganic constituents. It is assumed that the biological activities of selenium are dependent on the speciation of its metabolites, which are derived from the ingested selenium. In order to investigate the metabolism of various dietary forms of selenium, XANES was performed at Se K-edge absorption on bulk human lung cells exposed in vitro to three selenium compounds, selenomethionine, methylselenocystein, and selenite [82]. Using XANES LCF of ten reference selenium compounds, this study indicated that selenomethionine and methylselenocystein treated cells displayed distinct speciation profiles (Figure 8). In the selenomethionine treated cells selenium is found dominantly as $\mathrm{R}-\mathrm{Se}-\mathrm{R}$, characteristic of in organic selenium metabolites. In methylselenocystein treated cell, R-Se-R species constituted $80 \%-85 \%$ of the $\mathrm{Se}$, with the remaining $15 \%-20 \%$ composed of a diselenide species ( $R-S e-S e-R)$. Such difference between selenium diets was then confirmed by EXAFS investigation. Indeed, in the spectrum from methylselenocystein treated cells, a peak at $2.36 \AA$ in the Fourier transforms was only modeled with the addition of a Se scatterer to the fit, indicating clearly the diselenide as a product of intracellular metabolism of methylselenocystein. In contrast, the EXAFS spectrum of selenomethionine treated cells was readily fit by only two $\mathrm{C}$ scatterers with a Se-C bond length of $1.95 \AA$. Therefore, the comparison of the EXAFS and LCF XANES analysis of methylselenocystein- and selenomethionine-treated cells evidenced the presence of a diselenide in the methylselenocystein-treated cells only. The presence of diselenide species suggests that methylselenocystein generates metabolites that alter the redox balance of the cell to a more oxidizing environment than after selenomethionine treatment. In selenite treated cells mostly inorganic species are observed, together with $\mathrm{S}-\mathrm{Se}-\mathrm{S}$ and $\mathrm{R}-\mathrm{Se}-\mathrm{Se}-\mathrm{R}$ suggesting that selenite follows the same reductive metabolism pathway as elemental selenium. 


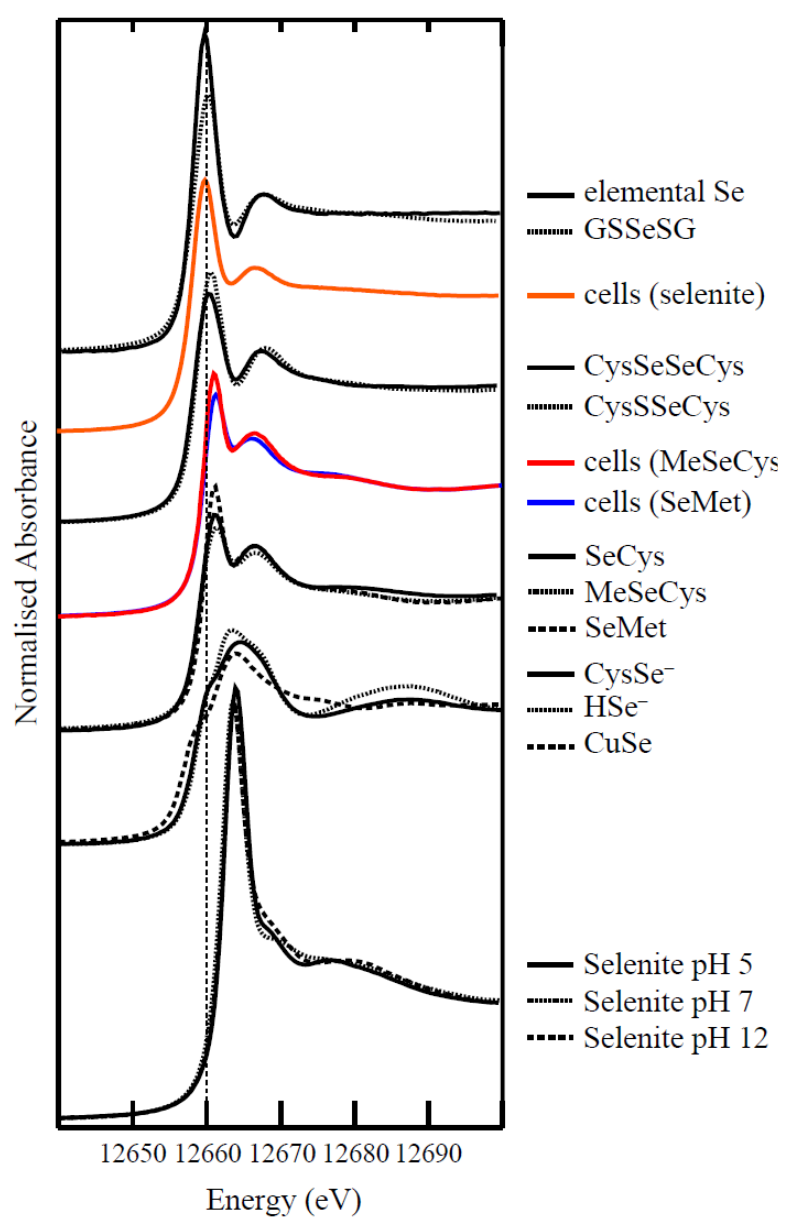

Fig. 8. Selenium K-edge absorption spectra of model selenium compounds used in the LCF of experimental spectra and examples of XANES spectra of cells treated with organic selenium compounds (blue and red) and inorganic selenite (orange). Adapted from [82].

Iron is suspected to be involved in tumoral progression by accelerating cellular proliferation. In colon cancer cells cultured in vitro and exposed to different chemical forms of Fe, inorganic $\mathrm{Fe}(\mathrm{II})$, inorganic Fe(III), transferrin and Fe-citrate, XANES speciation revealed that Fe was always present as $\mathrm{Fe}(\mathrm{III})$ in the cells, for all types of Fe exposure conditions [83]. XANES spectra were fitted using a combination of inorganic Fe(III) and transferrin and showed that when cells were exposed to inorganic $\mathrm{Fe}$, this element was stored in a non-ferritin like compartment. In this study, XANES analyses at Fe Kedge also enabled to distinguish between two cell lines with different rates of growth, low and high proliferation rates.

\subsection{Speciation of essential elements in neuropathology}

As previously discussed, XANES has proven to be a very powerful analytical tool for the speciation of sulfur chemical species in biological tissues, especially in brain tissues. XANES enables the speciation of sulfur in its native state by direct in situ analysis if appropriate protocols are applied. The article from Hackett et al. [30] has paved the way to sulfur speciation studies in neurosciences but also in other fields of physiopathology research as illustrated by the articles cited in the previous sections. In this initial work performed at the Stanford Synchrotron Radiation Lightsource, two types of brain compartments were compared, cerebellum and brain stem tissue sections from healthy male Sprague-Dawley rats. The results show significantly higher thiol, thio-ether, and sulfonic acid, and 
lower sulfate content within the cerebellum, relative to the brain stem. This study also demonstrated the possibility to detect markers of peroxidative stress, with a substantial increase in the level of sulfoxides observed following a corresponding decrease in the relative composition of thiols, after incubation of tissue sections with Fe(II). This study highlighted the relevance of $\mathrm{S}$ speciation using XANES at K-edge for neuropathological studies, where questions concerning free radical-mediated tissue damage could be addressed. This method was then applied by the same team to investigate global brain ischemia in animal models $[84,85]$. In these recent studies XANES speciation of sulfur was nicely combined to FTIR (Fourier Transform Infrared Spectroscopy) analysis on the same tissues sections to investigate at the same time protein aggregation. Increased disulfide levels, a direct marker of oxidative stress, and protein aggregation were observed after ischemia concomitantly to the loss of neuron integrity.

Dysfunction in the homeostatic control of essential metals in neurons has been associated to the etiology of various neurodegenerative disorders such as Alzheimer's, Parkinson's and Charcot's diseases, to cite only a few of the most relevant ones. XAS and more specifically micro-XANES have been successfully applied to investigate whether the changes in trace metals homeostasis could impair the redox status of these metals in neurons. Fe and $\mathrm{Cu}$ are the most widely studied metals in this field because of their redox chemistry and potential implication in the etiology of degenerative diseases through enhancement of oxidative stress. As it will be discussed in the following literature review, Fe is usually present as $\mathrm{Fe}(\mathrm{III})$ in brain and neurons, and modifications of Fe redox balance have not been observed yet in pathological conditions, although reduction to $\mathrm{Fe}(\mathrm{II})$ could be expected. In an animal model of Alzheimer's disease (AD), the oxidation state of Fe and $\mathrm{Cu}$ were determined by their Kabsorption edges as performed at the Beijing Synchrotron Radiation Facility [86]. This study showed that in an Alzheimer's disease animal model, Fe was present exclusively as Fe(III), most probably complexed to ferritin, and $\mathrm{Cu}$ as a mixture of $\mathrm{Cu}(\mathrm{I})$ and $\mathrm{Cu}(\mathrm{II})$. Unfortunately in this study the XANES data obtained on the Alzheimer's disease model were not compared to control animals, or to the stage of progression of the disease in such model. In a similar study on mouse models of AD performed at National Synchrotron Light Source, at Fe K-edge, no modification of Fe redox state could be observed in amyloid plaques between $A D$ model animals and controls, although the concentration of $\mathrm{Fe}$ increased in the AD model mice [87]. LCF of XANES spectra using Fe proteins as standards (ferritin, myoglobin, cytochrome $\mathrm{c}$, magnetite, and ferredoxin) suggested that Fe could be likely present as a combination of ferritin and myoglobin. As reviewed in 2014, XANES speciation of Fe oxidation state in samples either from human biopsies or from animal and cellular models of neurodegenerative diseases always evidenced Fe(III) species, without any change in redox state [8]. This trend is confirmed by a recent article applying Fe speciation using XANES on multiple sclerosis human brain tissues and showing again only Fe(III) species [88]. Most interestingly, in this late study, some differences among $\mathrm{Fe}$ (III) species were observed between brain regions, consisting of different proportions of iron oxide polymorphs (ferrihydrite, goethite, magnetite) and heme proteins, with for example a higher proportion of goethite in brain regions with high-iron. Goethite is the predominant iron biomineralization component of haemosiderin in thalassaemia and is usually not found in healthy brain tissues.

Cu speciation by XANES is usually more difficult to perform than Fe speciation in human tissues, because $\mathrm{Cu}$ concentrations are about one order of magnitude lower than $\mathrm{Fe}$, and close to the limit of speciation analysis using XANES. However in the brain, the $\mathrm{Cu}$ content can be locally enriched, enabling $\mathrm{Cu}$ speciation. This is the case of glial cells of the subventricular zone which have local $\mathrm{Cu}$ concentrations thousands of time higher than other cells [89]. In this study, XANES Cu K-edge was performed under cryogenic conditions to ensure non damaging conditions, on brain tissue sections from Male Sprague-Dawley rats (Figure 9). The result showed that in the Cu-rich region along the lateral ventricule wall, $\mathrm{Cu}$ is present as $\mathrm{Cu}(\mathrm{I})$ with a XANES spectrum very close to the $\mathrm{Cu}$ binding protein 
metallothionein, indicating that $\mathrm{Cu}$ is bound to thiols. This result has been confirmed in a further study using a metallothionein $(1,2)$ knock-out mouse model and showing the persistence of $\mathrm{Cu}_{x} \mathrm{~S}_{y}$ aggregates, suggesting that $\mathrm{Cu}$ is bound to metallothionein-3, or to a yet unknown protein, in astrocytes of the subventricular zone [90].

(A)

(B)

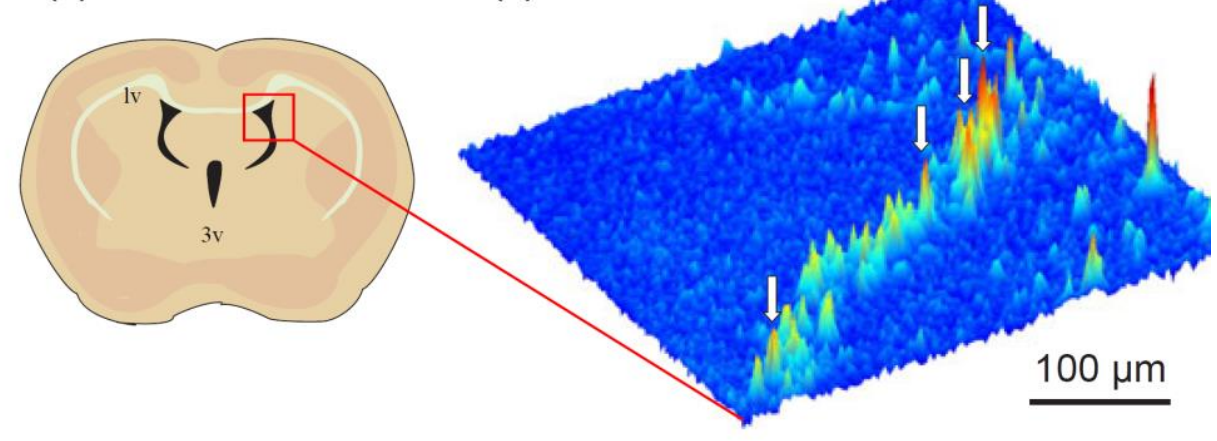

(C)

(D)
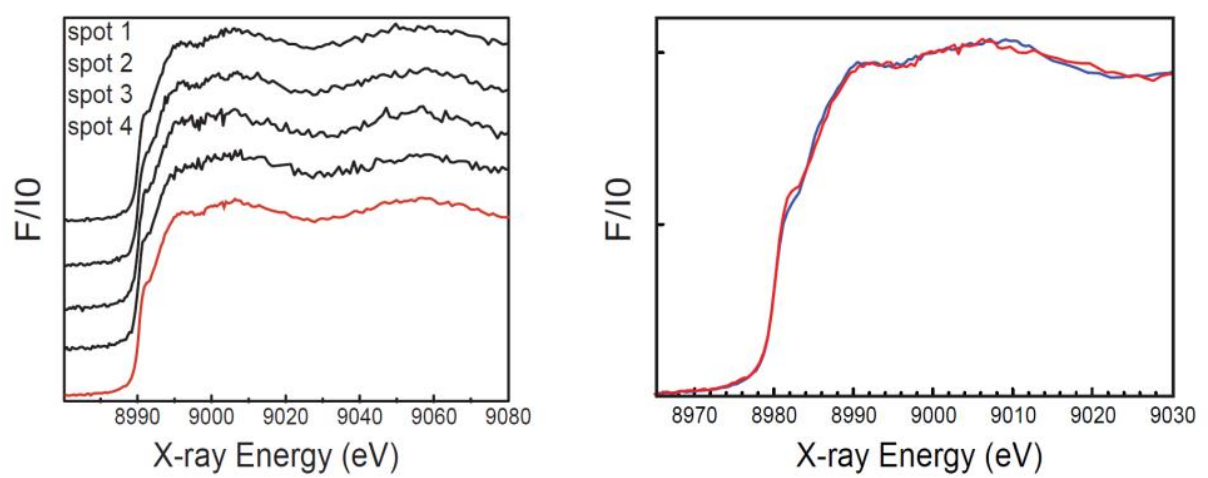

Fig. 9. (A) Schematic diagram of coronal brain section. The red box indicates the approximate area of the XRF image. (B) XRF image of copper distribution demonstrates that Cu enrichment is localized to a thin layer along the lateral ventricle wall. X-ray absorption near edge spectroscopy (XANES) measurements (C) were taken on $\mathrm{Cu}$ accumulations, indicated by arrows. Spectra obtained from different $\mathrm{Cu}$ accumulations (black line) and average of all XANES measurements (red line) taken on this sample. (D) Intracellular Cu K-edge XANES spectrum at low temperature on the $\mathrm{Cu}$ accumulations in a rodent brain (red) recorded at the same experimental conditions as the spectrum of $\mathrm{Cu}$ metallothionein prepared from rabbit apo-metallothionein (blue). Adapted from [89].

\section{Future trends}

\subsection{Hyphenated XAS}

The combination of XAS with chromatographic methods has probably been underused to date although it is a powerful complementary approach to the more conventional direct XAS speciation of the elements [91]. As previously discussed, one of the singularity of XAS compared to other biochemical characterization techniques is that it can directly be applied onto complex samples such as biological tissues. Such in situ spectroscopy is possible due to the specificity of the element absorption edge and the limited matrix effects, meaning that the speciation of one specific element can be performed in presence of all the other constituents of the sample. This represent both a strength and a drawback; a strength because XAS can be applied directly, almost without any sample 
preparation and any modification of the native speciation of the element; whilst a drawback because only the bulk speciation of a specific element is possible, meaning that if several chemical species coexist in the sample they will contribute all together to the XAS spectra. We have seen that by knowing the expected chemical species present in the sample it was possible to quantify their relative contributions by fitting the XAS spectrum to a linear sum of the individual spectra from each of the pure component. However this method has obviously some limitations, if all the initial components have not been pre-identified, if the contribution of some of the chemical species is low compared to others, etc. An elegant approach to counteract this drawback is to combine XAS to some chromatographic methods in order to separate the individual chemical species, but under an important condition, while maintaining the initial speciation state of the element using non-denaturating chromatographic methods such as native gel electrophoresis [92,93].

Despite the great potential of XAS hyphenated methods, there have been only few reports in the last years of XAS combination with chromatographic separation. However there is a great benefit to separate the biomolecules before element analysis in order to obtain specific information on element content and speciation from individual molecular species such as metalloproteins. Most of the studies so far have applied synchrotron XRF to the characterization of metalloproteins separated on electrophoresis gels, giving already valuable information about metal content in proteins [82,9498], but not about the chemical speciation. Gel electrophoresis separation prior to XAS concentrates metalloproteins in a narrow band of the gel and enables to perform not only XANES but also EXAFS, this late requiring higher quantities of elements to be applied successfully [28,99]. For example, it is possible to concentrate a single metalloprotein of interest from a crude protein extract and to study by EXAFS the metal coordination of this specific protein. This the case of the study published by Chevreux et al. [28], where EXAFS has been carried out on native IEF (Isoelectric Focusing Electrophoresis) gels at Zn K-edge to investigate the metallation state of SOD1 (superoxide dismutase), a $\mathrm{Cu}$ and $\mathrm{Zn}$ metalloprotein. This work depicts the protocols established for non-denaturating electrophoresis and subsequent XAS speciation. SOD1 is a ubiquitous antioxidant protein. Mutations of SOD1 are involved in familial cases of amyotrophic lateral sclerosis (ALS) and zinc-deficient mutant SOD1 has been hypothesized to be involved in the etiology of ALS. In this experiment performed at the ESRF, human wild type SOD1 and a pathological mutant, A4V, were separated from a crude cellular protein extract using native IEF. This protocol enabled not only to separate the SOD1 but also to identify various isoforms of SOD1 with different isoelectric points. It could be shown that the A4V mutant isoforms had higher isoelectric points than the wild type isoforms, that this shift towards more basic $\mathrm{pH}$ was accompanied by a decrease of the $\mathrm{Cu} / \mathrm{Zn}$ ratio of the protein but without any modification of the $\mathrm{Zn}$ coordination. EXAFS at Zn K-edge showed that atomic distances and Debye-Waller factors were in good agreement with published EXAFS analysis of SOD1 in solution or from crystals. For both the wild type and the mutant proteins, and for all the isoelectric point isoforms studied, the EXAFS data did not differ, suggesting that the mutant protein exhibits correctly structured Zn-binding sites. Interestingly, FAME beamline at ESRF enabled a low detection limit, as low as $10 \mu \mathrm{g} \cdot \mathrm{g}^{-1}$, to performed EXAFS. One of the limitations of this study is that it can only be applied to SOD proteins since the position of the protein on the gel is given by a colorimetric reaction for superoxide dismutase activity (Nitro blue tetrazolium staining). The other limitation is due to the one-dimensional separation electrophoresis that was adapted to SOD1 investigation with a yeast transgenic model that overexpresses the human SOD1, but of limited applicability to other samples.

To enlarge the field of application of XAS to any metalloproteins the use of $2 \mathrm{D}$ gel electrophoresis and western blot has been proposed and validated on standard metalloproteins [100]. To validate the methodology, commercially available metalloproteins, carbonic anhydrase, Cu,Zn SOD, and metallothionein have been separated by native 2D PAGE (Polyacrylamide Gel Electrophoresis) and blotted on a PVDF (Polyvinylidene difluoride) membrane. XANES at zinc K-edge was carried out on the 
PVDF membranes with carbonic anhydrase or metallothionein, whereas the full EXAFS spectrum could be collected for $\mathrm{Cu}, \mathrm{Zn} \mathrm{SOD}$. Although the method has not yet been applied to complex protein extracts, this article emphasizes the potential capability of hyphenated XAS for metal speciation in isolated proteins. It also demonstrated for the first time that western blot with PVDF membranes was suited for XAS analysis.

\subsection{In situ correlative imaging and speciation}

Similarly to the advantages of combining XAS together with chromatographic methods, microXAS and XAS imaging studies can greatly benefit from other imaging methods, performed before or concomitantly. When spatially resolved XAS is employed, other microscopic modalities are required to determine either morphologically or chemically the areas of interest on the sample. Micro-XAS is almost systematically performed together with micro-XRF to identify the region of interest, i.e. where to perform the point XAS of a specific element $[26,101]$. However the chemical element distribution as obtained by micro-XRF lacks precise morphological information which can be easily obtained using optical microscopy. This is particularly true for single cell analysis, the fluorescence labelling of biomolecules or organelles prior to micro-XAS and micro-XRF is highly beneficial since it enables identifying accurately the location of element species and those of labeled biomolecules [5, 32]. For example, using confocal microscopy of organelle's specific fluorescent probes, the oxidation state of arsenic could be determined by micro-XANES in the cytoplasm, the mitochondrial network and the nucleus of hepatocellular carcinoma single cells [102]. A similar approach was employed to identify the intracellular site of accumulation of $\mathrm{Mn}$ in mammalian cells and revealed that $\mathrm{Mn}$ was present at $\mathrm{Mn}$ (II) oxidation state in the Golgi apparatus and in the cytoplasm of dopaminergic cells [53]. Despite the real benefit of performing live cell fluorescence imaging prior to XAS in order to identify with accuracy cellular organelles, or fluorescent biomolecules, this approach has not yet been extensively employed.

\subsection{In vivo XANES}

XAS speciation analysis of living organisms is in practice very difficult to achieve due to beam damage and water radiolysis that rapidly alter the organism viability. Although it means that XAS is most often performed ex vivo, on biological tissue sections and biopsies it is feasible in some favorable cases. Invertebrates such as insects and plants can survive quite extended X-ray exposure with no apparent short-term effects [9]. Synchrotron-based speciation is for instance possible for plant samples. Living plants can be placed under the X-ray beam for in situ chemical speciation analysis as illustrated for selenium speciation by chemical specific XRF imaging, or by local micro-XANES [21,103]. Closer to biomedical applications, bulk XAS of sulfur speciation could be successfully carried out on living mammalian cells by maintaining the cells alive in the culture medium during the analysis using a specifically designed sample holder [104]. To limit beam damage the sample holder was translated to different positions between each energy scan.

Recently, micro-XRF and micro-XANES measurements were performed on a living hydrated organism, the nematode worm model Caenorhabditis elegans [105]. In vivo XAS presents many practical advantages. Indeed, due to the absence of any kind of cryogenic or chemical fixation steps, sample examination can be carried out in real physiological condition. Furthermore, a drastic time reduction and an overall experiment simplification are also obtained. However, in order to achieve a reliable result, several important key steps have to be adopted before the XAS measurements. First of all, in order to keep the sample static during the measurements, the nematodes were properly anesthetized and deposited in between two $\mathrm{Si}_{3} \mathrm{~N}_{4}$ membrane layers. Secondly, an accurate calibration 
of the radiation exposure had to be performed. Over the threshold limit of $10^{7} \mathrm{~Gy}$ dose many metals can undergo redox reactions at room temperature. The authors tested two irradiation setup conditions: called 'High Dose' ( $\left.10^{8} \mathrm{~Gy}\right)$ and 'Low Dose' $\left(10^{6} \mathrm{~Gy}\right)$, respectively. The samples were measured a second time after $5 \mathrm{~h}$ and a statistic correlation based on pixel analysis map was reported. Although 'Low Dose' irradiation sacrificed some spatial detail, up to a 10 factor in resolution, compared to 'High Dose' setup, it minimized photoreduction of metals but without losing much morphological information despite the alteration in spatial resolution. Once the photoreduction issue was solved, in order to prove their point, James and his group examined a combined genetic and exogenously challenged model of severe iron dyshomeostasis in C. elegans. Nematodes lacking the iron-storage protein ferritin were exposed to normal and iron rich media. As reported in the Figure 10, the precise energy of the Fe K pre-edge peak (around $7114 \mathrm{eV}$ ) arising from $1 \mathrm{~s} \rightarrow 3 \mathrm{~d}$, electronic transitions, reflects the relative abundance of ferrous Fe(II) and ferric Fe(III) iron. Interestingly, comparing the mean of each pixel XANES spectra for each measured individual, the centroid peak reduced intensity for the pre-edge transition in wild type $C$. elegans cultured on high Fe was suggestive of an increase in the number of octahedral Fe(III) centers, consistent with coordination geometry increased buffering of Fe within ferritin. Ferritin depleted $C$. elegans samples, regardless of Fe load, demonstrated a shift to lower centroid peak energies, indicating increased Fe(II) [105].

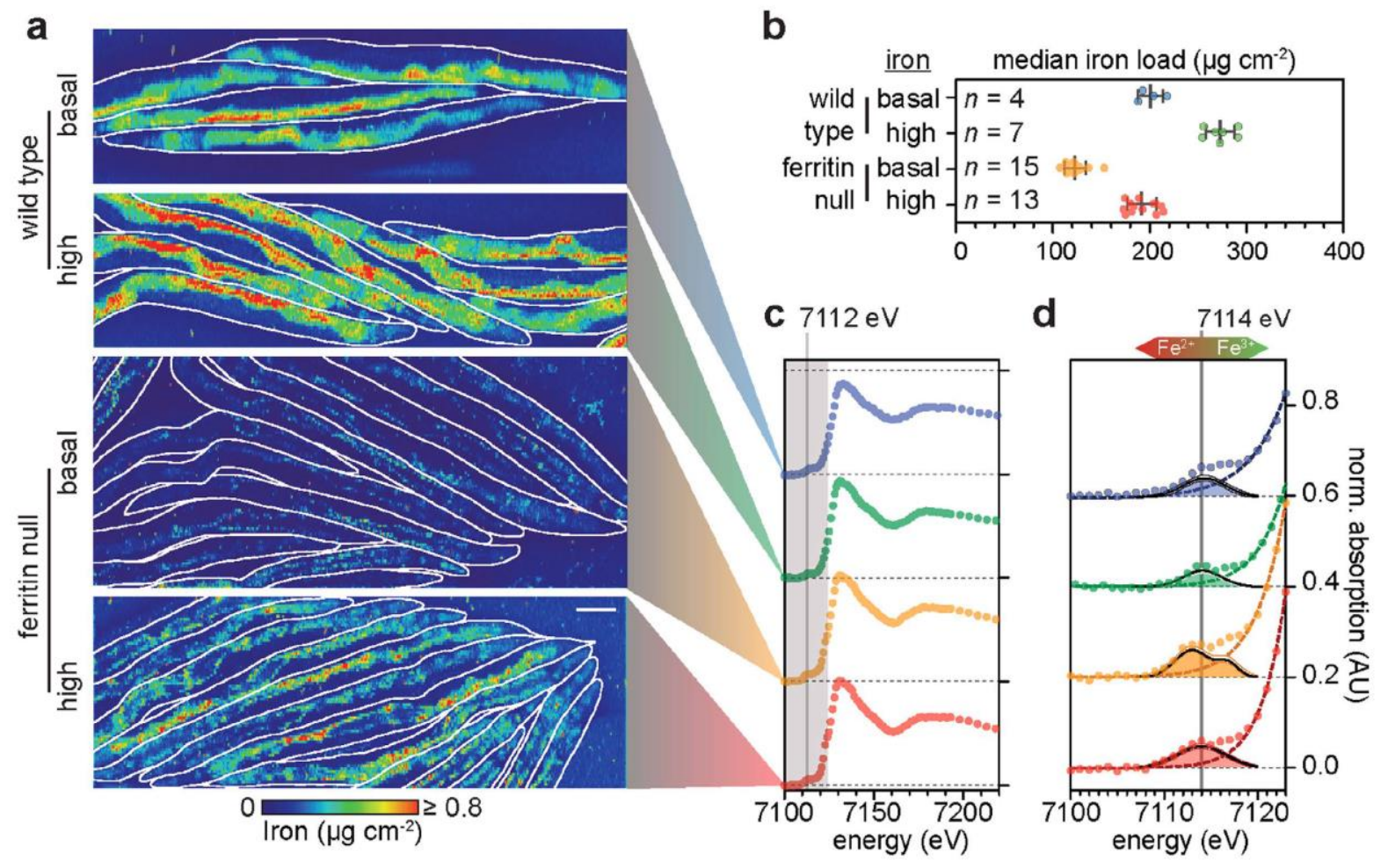

Fig. 10. (a) SXRF imaging of $C$. elegans wild type and ferritin nulls at basal and high Fe exposure. Scale bar $=100 \mu \mathrm{m}$. (b) Median iron areal density for each specimen (c) Iron XANES spectra (average of all pixels) extracted for the groups shown in (a). The starting position of Fe K-edge $(7112 \mathrm{eV})$ is marked with a vertical line. (d) Expanding the pre-edge region (grey box in (c)), following subtraction of the rising edge (dashed line), highlights changes in both the energy and intensity of the $1 \mathrm{~s} \rightarrow 3 \mathrm{~d}$ pre-edge feature between groups. The extracted data (colored circles) and fitted Gaussian are superimposed to determine the centroid values ( $\sim 7114 \mathrm{eV}$ for wild type; marked for reference). Loss of ferritin changed the pre-edge feature to exhibit two centroid energies $(7113 \mathrm{eV}$ and $7117 \mathrm{eV})$, whereas high iron exposure retained a single centroid energy of $7114 \mathrm{eV}$. Adapted from [105]. 
The results showed that Fe speciation was unchanged during in vivo analysis. The in vivo XANES alternative is simpler to perform than fully cryogenic analyses since it does not require a cryogenic sample stage but if a cryostage is available cryogenic analysis of frozen hydrated samples is preferred, especially for the speciation of elements with unstable chemical species or at low concentrations, and for samples less resistant to irradiation than the invertebrate $C$. elegans.

\subsection{Full Field XANES imaging}

FF XANES is a particular XAS acquisition mode in which an energetically tunable unfocused beam passes through the sample. The transmitted $X$-rays are subsequently converted into visible light by a scintillator crystal which is then collected by a CCD camera. The major advantages of FF XANES lie in the fast image acquisition, its micrometric resolution and large field of view, in the millimeter range. FF XANES is operated with a broad X-ray beam in transmission mode which in consequence limits its sensitivity compared to fluorescence mode micro-XANES. The spatial resolution is also limited by the diffraction limit of visible light to about $1 \mu \mathrm{m}$. Similarly to bulk XAS in transmission mode the sample thickness must be optimized in order to reduce the self-absorption while maintaining a sufficient thickness containing enough analyte. As consequence, FF XANES is applied to thin samples and for the chemical imaging of locally concentrated elements although recent progresses in detection systems have enlarged the capabilities of FF XANES applications [106]. Although FF XANES features are more adapted to geology, cultural heritage, or material sciences investigation, there are also extremely suitable for some specific areas of biomedical research such as the biomineralization studies. Hesse and coworkers recently exploited FF XANES to investigate the nature of human bone tissues, using FF XANES at Ca K-edge to enable detailed analysis of a single osteon [107]. Such analysis revealed that the white line feature relying on the intensity ratio between $1 \mathrm{~s}$ and $4 p$ dipole transitions, varied with respect to the lamellar orientation within the same osteon. This variation could be ascribed to polarization effects. Moreover, the dominant spectral features related to crystal orientation effects could also be obtained by collecting maps at only few specific energies instead of collecting XANES at hundreds of energy points as it is normally done. Interestingly, Hesse's work proved that light polarization dependency could be exploited to assess apatite orientations in bone tissue.

\subsection{X-ray Free Electron Laser XAS}

The state of the art in X-ray spectroscopy is represented by X-ray Free Electron Laser (XFEL). XFEL is at the moment the brightest tunable source available for spectroscopy studies [108]. The femtosecond impulse nature of such radiation makes it extremely suitable for time resolved studies on biologically active metallic centers. The XFEL rise is still at the beginning but supported by a fast growing community. In this context a complete review concerning the most biochemical relevant papers is offered by Kern and collaborators [109]. Despite most of the works rely on XFEL exploitation for crystallographic studies, recently also XAS and X-ray Emission Spectroscopy (XES) applications have been reported $[109,110]$. As demonstrated by Alonso, Kern and their groups, two are the main advantage of XFEL-XAS. Firstly, the beam pulse is so intense and rapid that the sample is probed before any photo-damage. As a consequence, there is no need of cryogenic conditions since the samples can be measured in their native form. However, it is important to stress that the biggest advantage is also the most limiting issue: indeed, the beam is so energetic that the sample is destroyed after each measurement. Secondly, the femto-second time scale definitively pushes further the limits of reaction 
process kinetics study compare to the pico-second time of the best conventional synchrotron XAS beamline $[109,110]$. In Alonso's paper, XFEL-XES were successful used for the first time to probe the charge and spin states as well as the ligand environment of $\mathrm{Mn}(\mathrm{II})$ and binuclear $\mathrm{Mn}_{2}(\mathrm{III}-\mathrm{IV})$ complexes at room temperature. The obtained K $\beta 1,3$ XES spectra were found in agreement with undamaged spectra collected at low dose using synchrotron radiation [110]. Interestingly, as shown by Kern and coworkers, XAS measurement by means of XFEL source can be exploited to probe the $3 \mathrm{~d}$ transition metal L-edge without the typically required ultrahigh vacuum setup. Noticeably, a novel hightransmission zone-plate spectrometer allowed $\mathrm{Mn}$ L-edge discrimination from the overwhelming $\mathrm{O} \mathrm{K}$ edge background [111]. By measuring partial fluorescence yield XAS, the authors provided information on the oxidation states, symmetry, and covalency of a dilute $\mathrm{Mn}$ aqueous solution suggesting applications of this system for studying metalloenzymes. Overall, sample destruction is the main limitation of XFEL XAS for biological applications development, dramatically limiting any X-ray imaging combined approach. However, the ultra-short femtosecond X-ray pulses could enable the speciation of transient oxidation states in redox reactions that cannot be addressed with synchrotron radiation X-ray sources.

\section{Conclusion}

Synchrotron-based speciation with XAS offers quite unique features for the analysis of chemical element species in biological samples. XAS is a noninvasive method of speciation that can be applied in situ. The increasing number of studies using XAS follows a general tendency in Analytical Chemistry with a shift from employing indirect methods of characterization to direct analytical methods requiring limited sample preparation and preserving the initial state of the sample. XAS is well adapted to the speciation of native chemical species, if applied with adequate protocols. It potentially avoids artifacts due to speciation modification consecutive to the sample processing used for indirect methods, such as homogenizing, heating, solubilizing, etc. In biological samples, because of the dilution of the elements, XANES is more often employed than EXAFS which requires higher element concentrations.

One of the main limitations however for such direct speciation analysis is that XAS measures concomitantly all the chemical element species present in the probed volume, often resulting in complex spectra. If the nature of the individual chemical species in presence is known, then the deconvolution of the XAS spectrum, most usually XANES, into the sum of the individual constituents is possible with LCF ad PCA approaches. Importantly this data analysis can be quantitative resulting in the determination of the relative contribution of each of the single species. Therefore XANES is a powerful method for the qualitative detection of chemical species and their relative quantitative determination. However, XANES lacks the capability to identify precisely unknown compounds, only some indications about oxidation states and chemical bounds can give assess to the nature of the unknown species. Also, the quantification of the relative proportions of the single constituents relies on the assumption made on the expected chemical species used as reference compounds, which in some cases might lead to misleading results if the initial assumptions were not correct. XANES is also limited at the time to quantify low abundant chemical species, typically below $5-10 \%$ of the total species amount, then requiring other analytical approaches. As for other analytical issues, direct XAS speciation analysis should be performed together with other indirect methods of speciation. Indirect and direct speciation methods usually complement each other for the speciation of the same analyte.

As mentioned XAS selectivity is limited but can be improved at least by two analytical strategies. XAS can be coupled to chromatographic methods, resulting in the less complex mixtures of the chemical species, but attention must be paid to develop nondenaturating chromatographic 
conditions to maintain the native chemical species. The other alternative to gain in selectivity is the use of spatially resolved XAS in order to probe smaller and better identified areas of the sample with potentially lower numbers of chemical species. Spatially resolved XAS offers the additional possibility to correlate the speciation analysis with morphological or chemical information obtained with other imaging methods such as optical fluorescence microscopy or X-ray fluorescence imaging.

XAS speciation has contributed significantly to the understanding of the biological transformation of chemical elements in biological tissues for a variety of biomedical investigations. XAS is an excellent tool to follow redox modifications of pharmaceutical compounds, toxic metals or NPs. It can help understanding the mechanism of action of metal-based pharmacological compounds, such as for example the bio-reductive activation of platinum(IV)-based anticancer drugs or their biotransformation in hypoxic tumors. XAS is an important and elegant method to assess the redox cycling of sulfur in biological samples with various applications in general physiology, oncology, and neurosciences. It is also very useful for the speciation analysis of selenium and arsenic, two elements with rich redox biochemistry and complex metabolization pathways. Sulfur, selenium and arsenic can undergo numerous biological transformations resulting in complex mixture of organic and inorganic species with a variety of oxidation states. For sulfur, selenium and arsenic as well as for some other elements such as $\mathrm{Cr}$, micro-XRF chemical specific imaging can be performed giving the full image of the chemical species distribution. The determination of redox states of biological important metals such as $\mathrm{Mn}, \mathrm{Fe}$, and $\mathrm{Cu}$ has also a great interest to understand the etiological mechanisms of major diseases such as cancer and neurodegenerative disorders. XAS has also proven to be a valuable tool to identify not only element oxidations states but also chemical phases such as the ones from calcium calcifications or uranium solid precipitates in cells. In the fast growing field of nanotoxicology, XAS techniques provided a large amount of data on NPs speciation and in particular on the fundamental solubility-toxicology dualism. However, it must be pointed out, that we are still far from filling the gap concerning the nanostructure behavior inside the cells since the spatial resolution of XAS is at best in the $100 \mathrm{~nm}$ level.

Perspectives in technical improvements will render XAS speciation even more attractive for biomedical applications. Improvements in spatial resolution, below $100 \mathrm{~nm}$, may open new fields of application in nanobiotechnology. Improvements in the detection systems and data acquisition will enable reduction in the radiation damage and better assessment of speciation at lower concentrations. Such developments are under progress, or are already available in some newly developed facilities. Improved detection and acquisition capabilities will enable an improved use of XANES imaging and FF XANES imaging for biomedical applications. XAS with crystal analyzer spectrometers already allows performing XANES down to $1 \mu \mathrm{g} \cdot \mathrm{g}^{-1}$ and in EXAFS down to $10 \mu \mathrm{g} \cdot \mathrm{g}^{-1}$, or lower. Although the objective to study living organisms seems highly challenging, in vivo XANES could also benefit from these technical developments enabling us to follow speciation and biotransformation in living organisms. FF XANES imaging enables to induce less irradiation damage and to perform rapid imaging of millimeter size samples at high spatial resolution having a great potential for biomineralization studies. Rapid, transient changes of redox states, such as those involved in metal redox cycling in living cells, can hardly be investigated by XAS to date but the development of XFEL may open new fields of research to study such rapid changes of chemical changes.

As a general conclusion, it is noteworthy that the number and the quality of publications in medical journals presenting XAS results are increasing. This trend is hopefully to be consolidated. To be successful, XAS studies require interdisciplinary work in close collaboration between physicist from synchrotron facilities, analytical chemists to develop reliable protocols, and biologists or medical doctors. This will be the recipe for increasing success of the method. As a long term perspective, the use of synchrotron radiation facilities to perform XAS not only for research purposes but also for medical diagnostics will probably be, in the future, a subject of discussion. 


\section{Acknowledgements}

The authors greatly acknowledge B. Trist for revising the manuscript and G. Clounez for providing coffee in abundance especially during the last days of writing.

\section{References}

[1] D.M. Templeton, F. Ariese, R. Cornelis, L.-G. Danielsson, H. Muntau, H.P. van Leeuwen, R. Lobinski, IUPAC Guidelines for Terms Related to Speciation of Trace Elements, Pure Appl. Chem. 72 (2000) 14531470.

[2] G. Bunker, Introduction to XAFS: A practical guide to X-ray Absorption Fine Structure Spectroscopy, Cambridge University Press, Cambridge, 2010.

[3] M. Newville, Fundamental of XAFS, Rev. Mineral. Geochem. 78 (2014) 33-74.

[4] M. Benfatto, C. Meneghini, A Close Look into the Low Energy Region of the XAS Spectra: The XANES Region, in: F. Boscherini, C. Meneghini, S. Mobilio (Eds.), Synchrotron Radiation: Basics, Methods and Applications, Springer-Verlag, Berlin-Heidelberg, 2015, pp. 213-240.

[5] R. Ortega, Direct speciation analysis of inorganic elements in single cells using X-ray absorption spectroscopy, J. Anal. At. Spectrom. 26 (2011) 23-29.

[6] R. Ortega, A. Carmona, I. Llorens, P.L. Solari, X-ray absorption spectroscopy of biological samples. A tutorial, J. Anal. At. Spectrom. 27 (2012) 2054-2065.

[7] A.A Hummer, A. Rompel, X-ray absorption spectroscopy: a tool to investigate the local structure of metal-based anticancer compounds in vivo, Adv Protein Chem Struct Biol. 93 (2013) 257-305.

[8] J.F. Collingwood, M.R. Davidson, The role of iron in neurodegenerative disorders: insights and opportunities with synchrotron light, Front Pharmacol. 5 (2014) doi: 10.3389/fphar.2014.00191.

[9] M.J. Pushie, I.J. Pickering, M. Korbas, M.J. Hackett, G.N. George, Elemental and chemically specific X-ray fluorescence Imaging of biological systems, Chem. Rev. 114 (2014) 8499-8541.

[10] M. Gräfe, E. Donner, R.N. Collins, E. Lombi, Speciation of metal(loid)s in environmental samples by X-ray absorption spectroscopy: A critical review, Anal. Chim. Acta 822 (2014) 1-22

[11] F.J. Zhao, K.L. Moore, E. Lombi, Y.G. Zhu, Imaging element distribution and speciation in plant cells, Trends Plant Sci. 19 (2014) 183-192.

[12] P.L. Hagedoorn, Microbial Metalloproteomics, Proteomes 3 (2015) 424-439.

[13] H.A. Castillo-Michel, C. Larue, A.E. Pradas Del Real, M. Cotte, G. Sarret, Practical review on the use of synchrotron based micro- and nano- X-ray fluorescence mapping and X-ray absorption spectroscopy to investigate the interactions between plants and engineered nanomaterials, Plant Physiol Biochem. 110 (2017) 13-32.

[14] J.E. Penner-Hahn, Characterization of "spectroscopically quiet" metals in biology, Coord Chem Rev. 249 (2005) 161-177. 
[15] J.J. Cotelesage, M.J. Pushie, P. Grochulski, I.J. Pickering, G.N. George, Metalloprotein active site structure determination: synergy between X-ray absorption spectroscopy and X-ray crystallography, J Inorg Biochem. 115 (2012) 127-137

[16] S.E.J. Bowman, J. Bridwell-Rabb, C.L. Drennan, Metalloprotein crystallography: more than a structure, Acc. Chem. Res. 49 (2016) 695-702

[17] S. D. Kelly, D. Hesterberg, B. Ravel, Analysis of Soils and Minerals Using X-ray Absorption Spectroscopy Soil Science Society of America, in: A.L. Ulery, L.R. Drees(Eds.), Soil Methods of Soil Analysis. Part 5. Mineralogical Methods, Soil Science Society of America, Madison, 2009, pp. 387-463.

[18] J.J. Rehr, R.C. Albers, Theoretical approaches to x-ray absorption fine structure, Rev. Mod. Phys. 72 (2000) 621-654.

[19] Y. Joly, X-ray absorption near edge structure calculations beyond the muffin-tin approximation, Phys. Rev. B 63 (2001) 125120-125129.

[20] M. Benfatto, S. Della Longa, C.R. Natoli, The MXAN procedure: a new method for analysing the XANES spectra of metalloproteins to obtain structural quantitative information, J Synchrotron Radiat. 10 (2003) 51-57.

[21] I.J. Pickering, R.C. Prince, D.E. Salt, G.N. George, Quantitative, chemically specific imaging of selenium transformation in plants, Proc Natl Acad Sci U S A. 97 (2000) 10717-10722.

[22] R. Ortega, B. Fayard, M. Salomé, G. Devès, J. Susini, Chromium oxidation state imaging in mammalian cells exposed in vitro to soluble or particulate chromate compounds. Chem. Res. Toxicol 18 (2005) 1512-1519.

[23] I.J. Pickering, L. Gumaelius, H.H. Harris, R.C. Prince, G. Hirsch, J.A. Banks, D.E. Salt, G.N George, Localizing the biochemical transformations of arsenate in a hyperaccumulating fern, Environ Sci Technol. 40 (2006) 5010-5014.

[24] P.M. Kopittke, M.D. de Jonge, P. Wang, B.A. McKenna, E. Lombi, D.J. Paterson, D.L. Howard, S.A. James, K.M. Spiers, C.G. Ryan, A.A. Johnson, N.W. Menzies, Laterally resolved speciation of arsenic in roots of wheat and rice using fluorescence-XANES imaging, New Phytol. 201 (2014) 1251-1262.

[25] N.P. Edwards, A. van Veelen, J. Anné, P.L. Manning, U. Bergmann, W.I. Sellers, V.M. Egerton, D. Sokaras, R. Alonso-Mori, K. Wakamatsu, S. Ito, R.A. Wogelius, Elemental characterisation of melanin in feathers via synchrotron X-ray imaging and absorption spectroscopy, Sci Rep. 6 (2016) doi: $10.1038 /$ srep34002.

[26] T. Bacquart, G. Devès, A. Carmona, R. Tucoulou, S. Bohic, R. Ortega, Subcellular speciation analysis of trace element oxidation states using synchrotron radiation micro-X-ray absorption near edge structure, Anal. Chem. 79 (2007) 7353-7359.

[27] L. A. Currie, Limits for qualitative detection and quantitative determination. Application to radiochemistry, Anal. Chem. 40 (1968) 586-593.

[28] S. Chevreux, I. Llorens, P.L. Solari, S. Roudeau, G. Devès, A. Carmona, D. Testemale, J.L. Hazemann, R. Ortega, Coupling of native IEF and Extended X-ray Absorption Fine Structure to characterize zinc binding sites from pl isoforms of SOD1 and A4V pathogenic mutant, Electrophoresis 33 (2012) 12761281 
[29] O. Proux, E. Lahera, W. Del Net, I. Kieffer, M. Rovezzi, D. Testemale, M. Irar, S. Thomas, A. AguilarTapia, E.F. Bazarkina, A. Prat, M. Tella, M. Auffan, J. Rose, J. Hazemann, High-Energy Resolution Fluorescence Detected X-Ray Absorption Spectroscopy: A Powerful New Structural Tool in Environmental Biogeochemistry Sciences, J. Environ. Qual (2017) doi:10.2134/jeq2017.01.0023.

[30] M.J. Hackett, S.E. Smith, P.G Paterson, H. Nichol, I.J. Pickering, G.N. George, X-ray absorption spectroscopy at the sulfur K-edge: a new tool to investigate the biochemical mechanisms of neurodegeneration, ACS Chem. Neurosci. 3 (2012) 178-185.

[31] J. Chwiej, M. Szczerbowska-Boruchowska, M. Lankosz, S. Wojcik, G. Falkenberg, Z. Stegowski, Z. Setkowicz, Preparation of tissue samples for X-ray fluorescence microscopy, Spectrochim. Acta B 60 (2005) 1531-1537

[32] S. Roudeau, A. Carmona, L. Perrin, R. Ortega Correlative organelle fluorescence microscopy and synchrotron X-ray chemical element imaging in single cells, Anal. Bioanal. Chem 406 (2014) 6979-6991.

[33] L. Perrin, A. Carmona, S. Roudeau, R. Ortega, Evaluation of sample preparation methods for single cell quantitative element imaging using proton or synchrotron radiation focused beams, J. Anal. At. Spectrom. 30 (2015) 2525-2532.

[34] I. Hurbain, M. Saschse, The future is cold: cryo-preparation methods for transmission electron microscopy of cells, Biol. Cell 103 (2011) 405-420.

[35] G.N. George, I.J. Pickering, M.J. Pushie, K. Nienaber, M.J. Hackett, I. Ascone, B. Hedman, K.O. Hodgson, J.B. Aitken, A. Levina, C.Gloverf, P.A. Lay, X-ray-induced photo-chemistry and X-ray absorption spectroscopy of biological samples, J Synchrotron Rad. 19 (2012) 875-886.

[36] G. Veronesi, E. Koudouna, M. Cotte, F.L. Martin, A.J. Quantock, X-ray absorption near-edge structure (XANES) spectroscopy identifies differential sulfur speciation in corneal tissue, Anal Bioanal Chem. 405 (2013) 6613-6620.

[37] J. Canche-Tello, M.C. Vargas, J. Hérnandez-Cobos, I. Ortega-Blake, A. Leclercq, P.L. Solari, J. Lezama-Pacheco, C. Den Auwer, J. Mustre de Leon, X-ray accelerated photo-oxidation of As(III) in solution, J. Phys. Chem. A 119 (2015) 2829-2833.

[38] A.A. Hummer, A. Rompel, The use of X-ray absorption and synchrotron based micro-X-ray fluorescence spectroscopy to investigate anti-cancer metal compounds in vivo and in vitro, Metallomics 5 (2013) 597-614.

[39] M.D. Hall, H.L. Daly, J.Z. Zhang, M. Zhang, R.A. Alderden, D. Pursche, G.J. Foran, T.W. Hambley, Quantitative measurement of the reduction of platinum(IV) complexes using X-ray absorption nearedge spectroscopy (XANES), Metallomics 4 (2012) 568-575.

[40] C.K. Chen, J.Z. Zhang, J.B. Aitken, T.W. Hambley, Influence of equatorial and axial carboxylato ligands on the kinetic inertness of platinum(IV) complexes in the presence of ascorbate and cysteine and within DLD-1 cancer cells, J Med Chem. 56 (2013) 8757-8764.

[41] A.A. Hummer, P. Heffeter, W. Berger, M. Filipits, D. Batchelor, G.E. Büchel, M.A. Jakupec, B.K. Keppler, A. Rompel, X-ray absorption near edge structure spectroscopy to resolve the in vivo chemistry of the redox-active indazolium trans-[Tetrachlorobis(1H-indazole)ruthenate(III)] (KP1019), J Med Chem. 56 (2013) 1182-1196. 
[42] A. Levina, J.B. Aitken, Y.Y. Gwee, Z.J. Lim, M. Liu, A.M. Singharay, P.F. Wong, P.A. Lay, Biotransformations of anticancer ruthenium(III) complexes: an X-ray absorption spectroscopic study. Chemistry 19 (2013) 3609-3619.

[43] G.K. Gransbury, P. Kappen, C.J. Glover, J.N. Hughes, A. Levina, P.A. Lay, I.F. Musgrave, H.H. Harris Comparison of KP1019 and NAMI-A in tumour-mimetic environments. Metallomics 8 (2016) 762-773.

[44] A.A. Hummer, C. Bartel, V.B. Arion, M.A. Jakupec, W. Meyer-Klaucke, T. Geraki, P.D. Quinn, A. Mijovilovich, B.K. Keppler, A. Rompel, X-ray absorption spectroscopy of an investigational anticancer gallium(III) drug: interaction with serum proteins, elemental distribution pattern, and coordination of the compound in tissue, J Med Chem. (2012) 55 5601-5613.

[45] A. Levina, A.I. McLeod, P.A. Lay, Vanadium speciation by XANES spectroscopy: a three-dimensional approach, Chemistry 20 (2014) 12056-12060.

[46] C.M. Weekley, I. Kenkel, R. Lippert, S. Wei, D. Lieb, T. Cranwell, J.L. Wedding, A.S. Zillmann, R. Rohr, M.R. Filipovic, I. Ivanović-Burmazović, H.H. Harris, Cellular Fates of Manganese(II) Pentaazamacrocyclic Superoxide Dismutase (SOD) Mimetics: Fluorescently Labeled MnSOD Mimetics, X-ray Absorption Spectroscopy, and X-ray Fluorescence Microscopy Studies, Inorg Chem. 56 (2017) 6076-6093.

[47] A. Levina, A.I. McLeod, L.E. Kremer, J.B. Aitken, C.J. Glover, B. Johannessen, P.A. Lay, Reactivityactivity relationships of oral anti-diabetic vanadium complexes in gastrointestinal media: an $\mathrm{X}$-ray absorption spectroscopic study, Metallomics 6 (2014) 1880-1888.

[48] A. Levina, A.I. McLeod, S.J. Gasparini, A. Nguyen, W.G. De Silva, J.B. Aitken, H.H. Harris, C. Glover, B. Johannessen, P.A. Lay, Reactivity and Speciation of Anti-Diabetic Vanadium Complexes in Whole Blood and Its Components: The Important Role of Red Blood Cells, Inorg Chem. 54 (2015) 7753-7766.

[49] A.J. Hart, P.D. Quinn, F. Lali, B. Sampson, J.A. Skinner, J.J. Powell, J. Nolan, K. Tucker, S. Donell, A. Flanagan, J.F. Mosselmans, Cobalt from metal-on-metal hip replacements may be the clinically relevant active agent responsible for periprosthetic tissue reactions, Acta Biomater. 8 (2012) 38653873.

[50] T. Sugiyama, M. Uo, T. Wada, D. Omagari, K. Komiyama, T. Noguchi, Y. Jinbu, M. Kusama, Estimation of trace metal elements in oral mucosa specimens by using SR-XRF, PIXE, and XAFS, Biometals. 28 (2015) 11-20.

[51] K.M. Shah, P.D. Quinn, A. Gartland, J.M.J Wilkinson, Understanding the tissue effects of tribocorrosion: uptake, distribution, and speciation of cobalt and chromium in human bone cells, Orthop Res. 33 (2015) 114-121.

[52] L. Pascolo, A. Gianoncelli, G. Schneider, M. Salomé, M. Schneider, C. Calligaro, M. Kiskinova, M. Melato, C. Rizzardi, The interaction of asbestos and iron in lung tissue revealed by synchrotron-based scanning X-ray microscopy, Sci Rep. 3 (2013) doi: 10.1038/srep01123.

[53] A. Carmona, S. Roudeau, L. Perrin, G. Veronesi, R. Ortega, Environmental manganese compounds accumulate as $\mathrm{Mn}(\mathrm{II})$ within the Golgi apparatus of dopamine cells: relationship between speciation, subcellular distribution, and cytotoxicity, Metallomics 6 (2014) 822-832.

[54] V. Pierrefite-Carle, S. Santucci-Darmanin, V. Breuil, T. Gritsaenko, C. Vidaud, G. Creff, P.L. Solari, S. Pagnotta, R. Al-Sahlanee, C.D. Auwer, G.F. Carle, Effect of natural uranium on the UMR-106 
osteoblastic cell line: impairment of the autophagic process as an underlying mechanism of uranium toxicity, Arch Toxicol. 91 (2017) 1903-1914.

[55] Y. Zhu, X. Cai, J. Li, Z. Zhong, Q. Huang, C. Fan, Synchrotron-based X-ray microscopic studies for bioeffects of nanomaterials, Nanomedicine. 10 (2014) 515-524.

[56] Y.F. Li, J. Zhao, Y. Qu, Y. Gao, Z. Guo, Z. Liu, Y. Zhao, C. Chen, Synchrotron radiation techniques for nanotoxicology, Nanomedicine 11 (2015) 1531-1549.

[57] L. Wang, J. Li, J. Pan, X. Jiang, Y. Ji, Y. Li, Y. Qu, Y. Zhao, X. Wu, C. Chen, Revealing the binding structure of the protein corona on gold nanorods using synchrotron radiation-based techniques: understanding the reduced damage in cell membranes, J Am Chem Soc. 135 (2013) 17359-17368.

[58] L. Wang, T. Zhang, P. Li, W. Huang, J. Tang, P. Wang, J. Liu, Q. Yuan, R. Bai, B. Li, K. Zhang, Y. Zhao, C. Chen, Use of Synchrotron Radiation-Analytical Techniques To Reveal Chemical Origin of SilverNanoparticle Cytotoxicity, ACS Nano 9 (2015) 6532-6547.

[59] R.A. Davidson, D.S. Anderson, L.S. Van Winkle, K.E. Pinkerton, T. Guo, Evolution of silver nanoparticles in the rat lung investigated by X-ray absorption spectroscopy, J Phys Chem A. 119 (2015) 281-289.

[60] X. Jiang, T. Miclăuş, L. Wang, R. Foldbjerg, D.S. Sutherland, H. Autrup, C. Chen, C. Beer, Fast intracellular dissolution and persistent cellular uptake of silver nanoparticles in CHO-K1 cells: implication for cytotoxicity, Nanotoxicology 9 (2015) 181-189.

[61] S. Smulders, C. Larue, G. Sarret, H. Castillo-Michel, J. Vanoirbeek, P.H. Hoet Lung distribution, quantification, co-localization and speciation of silver nanoparticles after lung exposure in mice. Toxicol Lett. 238 (2015) 1-6.

[62] Veronesi G, Aude-Garcia C, Kieffer I, Gallon T, Delangle P, Herlin-Boime N, Rabilloud T, Carrière $\mathrm{M}$., Exposure-dependent $\mathrm{Ag}+$ release from silver nanoparticles and its complexation in AgS2 sites in primary murine macrophages, Nanoscale 7 (2015) 7323-7330.

[63] G. Veronesi, T. Gallon, A. Deniaud, B. Boff, C. Gateau, C. Lebrun, C. Vidaud, F. Rollin-Genetet, M. Carrière, I. Kieffer, E. Mintz, P. Delangle, I. Michaud-Soret, XAS Investigation of Silver(I) Coordination in Copper(I) Biological Binding Sites, Inorg Chem. 54 (2015) 11688-11696.

[64] G. Veronesi, A. Deniaud, T. Gallon, P.H. Jouneau, J. Villanova, P. Delangle, M. Carrière, I. Kieffer, P. Charbonnier, E. Mintz, I. Michaud-Soret, Visualization, quantification and coordination of Ag+ ions released from silver nanoparticles in hepatocytes, Nanoscale. 8 (2016) 17012-17021.

[65] M. Roman, C. Rigo, H. Castillo-Michel, I. Munivrana, V. Vindigni, I. Mičetić, F. Benetti, L. Manodori, W.R. Cairns, Hydrodynamic chromatography coupled to single-particle ICP-MS for the simultaneous characterization of AgNPs and determination of dissolved Ag in plasma and blood of burn patients, Anal Bioanal Chem. 408 (2016) 5109-5124.

[66] F. Tian, G. Chen, P. Yi, J. Zhang, A. Li, J. Zhang, L. Zheng, Z. Deng, Q. Shi, R. Peng, Q. Wang, Fates of Fe3O4 and Fe3O4@SiO2 nanoparticles in human mesenchymal stem cells assessed by synchrotron radiation-based techniques, Biomaterials. 35 (2014) 6412-6421.

[67] B. Wang, Q. Wang, H. Chen, X. Zhou, H. Wang, H. Wang, J. Zhang, W., Feng Size-Dependent Translocation Pattern, Chemical and Biological Transformation of Nano- and Submicron-Sized Ferric Oxide Particles in the Central Nervous System, J Nanosci Nanotechnol. 16 (2016) 5553-5561. 
[68] A. Ivask, K.G. Scheckel, P. Kapruwan, V. Stone, H. Yin, N.H. Voelcker, E. Lombi, Complete transformation of $\mathrm{ZnO}$ and $\mathrm{CuO}$ nanoparticles in culture medium and lymphocyte cells during toxicity testing, Nanotoxicology 11 (2017) 150-156.

[69] A. Buckley, J. Warren, A. Hodgson, T. Marczylo, K. Ignatyev, C. Guo, R. Smith, Slow lung clearance and limited translocation of four sizes of inhaled iridium nanoparticles, Part Fibre Toxicol. 10 (2017) doi: 10.1186/s12989-017-0185-5.

[70] A. Rompel, R.M. Cinco, M.J. Latimer, A.E. McDermott, R.D. Guiles, A. Quintanilha, R.M. Krauss, K. Sauer, V.K. Yachandra, M.P. Klein, Sulfur K-edge X-ray absorption spectroscopy: a spectroscopic tool to examine the redox state of S-containing metabolites in vivo, Proc. Natl. Acad. Sci. 95 (1998) 61226127.

[71] K. Xia, F. Weesner, W.F. Bleam, P.R. Bloom, U.L. Skyllberg, P.A. Helmke, XANES studies of oxidation states of sulfur in aquatic and soil humic substances, Soil Sci Soc Am J 62 (1998) 1240-1246.

[72] T.V. Mishanina, M. Libiad, R. Banerjee, Biogenesis of reactive sulfur species for signaling by hydrogen sulfide oxidation pathways, Nat Chem Biol. 11 (2015) 457-464.

[73] J. Prietzel, A. Botzaki, N. Tyufekchieva, M. Brettholle, J. Thieme, W. Klysubun, Sulfur speciation in soil by S K-edge XANES spectroscopy: comparison of spectral deconvolution and linear combination fitting, Environ Sci Technol 45 (2011) 2878-2886.

[74] E. Koudouna, G. Veronesi, I.I. Patel, M. Cotte, C. Knupp, F.L. Martin, A.J. Quantock, Chemical composition and sulfur speciation in bulk tissue by $\mathrm{x}$-ray spectroscopy and $\mathrm{x}$-ray microscopy: corneal development during embryogenesis, Biophys J. 103 (2012) 357-364.

[75] S.D. Blaschko, T. Chi, J. Miller, L. Flechner, S. Fakra, P. Kapahi, A. Kahn, M.L. Stoller, Strontium substitution for calcium in lithogenesis, J Urol. 189 (2013) 735-739

[76] D. Bazin, A. Dessombz, C. Nguyen, H.K. Ea, F. Lioté, J. Rehr, C. Chappard, S. Rouzière, D. Thiaudière, S. Reguer, M. Daudon, The status of strontium in biological apatites: an XANES/EXAFS investigation, J Synchrotron Radiat. 21 (2014) 136-42.

[77] A. Dessombz, C. Nguyen, H.K. Ea, S. Rouzière, E. Foy, D. Hannouche, S. Réguer, F.E. Picca, D. Thiaudière, F. Lioté, M. Daudon, D. Bazin, Combining $\mu X$-ray fluorescence, $\mu$ XANES and $\mu$ XRD to shed light on Zn2+ cations in cartilage and meniscus calcifications, J Trace Elem Med Biol. 27 (2013) 326333.

[78] M.J. Ceko, K. Hummitzsch, N. Hatzirodos, W. Bonner, S.A. James, J.K. Kirby, R.J. Rodgers, H.H. Harris, Distribution and speciation of bromine in mammalian tissue and fluids by $\mathrm{X}$-ray fluorescence imaging and X-ray absorption spectroscopy, Metallomics 7 (2015) 756-765

[79] O. Hachmöller, A.G. Buzanich, M. Aichler, M. Radtke, D. Dietrich, K. Schwamborn, L. Lutz, M. Werner, M. Sperling, A. Walch, U. Karst, Elemental bioimaging and speciation analysis for the investigation of Wilson's disease using $\mu$ XRF and XANES, Metallomics. 8 (2016) 648-653.

[80] M. Szczerbowska-Boruchowska, Z. Stegowski, M. Lankosz, M. Szpaka, D. Adamek, A synchrotron radiation micro-X-ray absorption near edge structure study of sulfur speciation in human brain tumors-a methodological approach, J. Anal. At. Spectrom. 27 (2012) 239-247.

[81] S. Kumar, X. Liu, F. Borondics, Q. Xiao, R. Feng, E. Goormaghtigh, F. Nikolajeff, Insights into Biochemical alteration in cancer-associated fibroblasts by using novel correlative spectroscopy, ChemistryOpen 6 (2017) 149-157. 
[82] C.M. Weekley, J.B. Aitken, L. Finney, S. Vogt, P.K. Witting, H.H. Harris, Selenium metabolism in cancer cells: the combined application of XAS and XFM techniques to the problem of selenium speciation in biological systems, Nutrients 5 (2013) 1734-1756.

[83] V.G. Mihucz, F. Meirer, Z. Polgári, A. Réti, G. Pepponi, D. Ingerle, N. Szoboszlai, C. Streli, Iron overload of human colon adenocarcinoma cells studied by synchrotron-based X-ray techniques, J Biol Inorg Chem. 21 (2016) 241-249.

[84] M.J. Hackett, S.E. Smith, S. Caine, H. Nichol, G.N. George, I.J. Pickering, P.G. Paterson, Novel biospectroscopic imaging reveals disturbed protein homeostasis and thiol redox with protein aggregation prior to hippocampal CA1 pyramidal neuron death induced by global brain ischemia in the rat, Free Radic. Biol. Med 89 (2015) 806-818.

[85] M.J. Hackett, C.J. Britz, P.G. Paterson, H. Nichol, I.J. Pickering, G.N. George, In situ biospectroscopic investigation of rapid ischemic and postmortem induced biochemical alterations in the rat brain, ACS Chem Neurosci. 6 (2015) 226-238.

[86] H. Wang, M. Wang, B. Wang, M. Li, H. Chen, X. Yu, Y. Zhao, W. Feng, Z. Chaia, The distribution profile and oxidation states of biometals in APP transgenic mouse brain: dyshomeostasis with age and as a function of the development of Alzheimer's disease, Metallomics 4 (2012) 289-296.

[87] M.W. Bourassa, A.C. Leskovjan, R.V. Tappero, E.R. Farquhar, C.A. Colton, W.E. Van Nostrand, L.M. Miller, Elevated copper in the amyloid plaques and iron in the cortex are observed in mouse models of Alzheimer's disease that exhibit neurodegeneration, Biomed Spectrosc Imaging. 2 (2013) 129-139.

[88] B.F. Popescu, J.M. Frischer, S.M. Webb, M. Tham, R.C. Adiele, C.A. Robinson, P.D. Fitz-Gibbon, S.D. Weigand, I. Metz, S. Nehzati, G.N. George, I.J. Pickering, W. Brück, S. Hametner, H. Lassmann, J.E. Parisi, G. Yong, C.F. Lucchinetti, Pathogenic implications of distinct patterns of iron and zinc in chronic MS lesions, Acta Neuropathol. (2017) 134 45-64.

[89] Y. Pushkar, G. Robison, B. Sullivan, S.X. Fu, M. Kohne, W. Jiang, S. Rohr, B. Lai, M.A. Marcus, T. Zakharova, W. Zheng, Aging results in copper accumulations in glial fibrillary acidic protein-positive cells in the subventricular zone, Aging Cell. 12 (2013) 823-832.

[90] B. Sullivan, G. Robison, J. Osborn, M. Kay, P. Thompson, K. Davis, T. Zakharova, O. Antipova, Y. Pushkar, On the nature of the Cu-rich aggregates in brain astrocytes, Redox Biol. 11 (2017) 231-239.

[91] R. Ortega, Synchrotron radiation for direct analysis of metalloproteins on electrophoresis gels, Metallomics 1 (2009) 137-141.

[92] S. Chevreux, S. Roudeau, A. Fraysse, A. Carmona, G. Devès, P.L. Solari, T.C. Weng, R. Ortega Direct speciation of metals in copper-zinc superoxide dismutase isoforms on electrophoresis gels using X-ray absorption near edge structure, J. Anal. At. Spectrom 23 (2008) 1117-1120.

[93] L. Finney, Y. Chishti, T. Khare, C. Giometti, A. Levina, P.A. Lay, S. Vogt, Imaging metals in proteins by combining electrophoresis with rapid x-ray fluorescence mapping, ACS Chem Biol. 5 (2010) 577587.

[94] S. Homma-Takeda, M. Shinyashiki, Y. Kumagai, N. A. Shimoto, new method for detection of mercury bound protein with a combination of gel electrophoresis and one dimensional synchrotron radiation X-ray fluorescence analysis, J Occup Health 38 (1996) 118-119. 
[95] Y. Gao, C. Chen, Z. Chai, J. Zhao, J. Liu, P. Zhang, W. He, Y. Huang, Detection of metalloproteins in human liver cytosol by synchrotron radiation X-ray fluorescence combined with gel filtration chromatography and isoelectric focusing separation, Analyst. 127 (2002) 1700-1704.

[96] M. Kühbacher, G. Weseloh, A. Thomzig, H. Bertelsmann, G. Falkenberg, M. Radtke, H. Riesemeier, A. Kyriakopoulos, M. Beekes, D. Behne, Analysis and localization of metal- and metalloid-containing proteins by synchrotron radiation x-ray fluorescence spectrometry, X-Ray Spectrom. 34 (2005) 112117.

[97] F.M. Verbi, S.C. Arruda, A.P. Rodriguez, C.A. Pérez, M.A. Arruda, Metal-binding proteins scanning and determination by combining gel electrophoresis, synchrotron radiation $\mathrm{X}$-ray fluorescence and atomic spectrometry, J Biochem Biophys Methods 62 (2005) 97-109.

[98] S. Matsuyama, A. Matsunaga, S. Sakamoto, Y. lida, Y. Suzuki, Y. Ishizaka, K. Yamauchi, T. Ishikawa, M. Shimura, Scanning protein analysis of electrofocusing gels using X-ray fluorescence, Metallomics 5 (2013) 492-500.

[99] Chevreux S., Solari P.L., Roudeau S., Devès G., Alliot I.,Testemale D., Hazemann J.L. Ortega R. (2009) EXAFS analysis of a human $\mathrm{Cu}, \mathrm{Zn}$ SOD isoform focusedusing non-denaturing gel electrophoresis. Journal of Physics IV, 190, 012205.

[100] J. Ward, E. Ollmann, E. Maxey, L.A., Finney X-ray absorption spectroscopy of metalloproteins, Methods Mol Biol. 1122 (2014) 171-87.

[101] L. Yang, R. McRae, M.M. Henary, R. Patel, B. Lai, S. Vogt, C.J. Fahrni, Imaging of the intracellular topography of copper with a fluorescent sensor and by synchrotron x-ray fluorescence microscopy. Proc Natl Acad Sci U S A. 102 (2005) 11179-11184.

[102] T. Bacquart, G. Devès, R. Ortega, Direct speciation analysis of arsenic in sub-cellular compartments using micro-X-ray absorption spectroscopy, Environ. Res. 110 (2010) 413-416.

[103] E. Bulska, I.A. Wysocka, M.L. Wierzbicka, K. Proost, K. Janssens, G. Falkenberg, In vivo investigation of the distribution and the local speciation of selenium in Allium cepa $L$. by means of microscopic X-ray absorption near-edge structure spectroscopy and confocal microscopic X-ray fluorescence analysis, Anal Chem. 78 (2006) 7616-7624.

[104] M. Gnida, E.Y. Sneeden, J.C. Whitin, R.C. Prince, I.J. Pickering, M. Korbas, G.N. George, Sulfur Xray absorption spectroscopy of living mammalian cells: an enabling tool for sulfur metabolomics. In situ observation of uptake of taurine into MDCK cell, Biochemistry 46 (2007) 14735-14741.

[105] S.A. James, D.J. Hare, N.L. Jenkins, M.D.de Jonge, A.I. Bush, G. McColl, фXANES: In vivo imaging of metal-protein coordination environments, Sci Rep. 6 (2016) doi: 10.1038/srep20350.

[106] P. Tack, J. Garrevoet, S. Bauters, B. Vekemans, B. Laforce, E. Van Ranst, D. Banerjee, A. Longo, W. Bras, L. Vincze, Full-field fluorescence mode micro-XANES imaging using a unique energy dispersive CCD detector, Anal Chem. 86 (2014) 8791-8797.

[107] B. Hesse, M. Salome, H. Castillo-Michel, M. Cotte, B. Fayard, C.J. Sahle, W. De Nolf, J. Hradilova, A. Masic, B. Kanngießer, M. Bohner, P. Varga, K. Raum, S. Schrof, Full-Field Calcium K-Edge X-ray Absorption Near-Edge Structure Spectroscopy on Cortical Bone at the Micron-Scale: Polarization Effects Reveal Mineral Orientation, Anal Chem. 88 (2016) 3826-3835. 
[108] T. Ishikawa, H. Aoyagi, T. Asaka, Y. Asano, N. Azumi, T. Bizen, H. Ego , K. Fukami, T. Fukui, Y. Furukawa, S. Goto, A compact X-ray free-electron laser emitting in the sub-angstrom region, Nat. Photon 6 (2012) 540-544.

[109] J. Kern, V.K. Yachandra, J. Yano, Metalloprotein structures at ambient conditions and in real-time: biological crystallography and spectroscopy using X-ray free electron lasers, Curr Opin Struct Biol. 34 (2015) 87-98.

[110] R. Alonso-Mori, J. Kern, R.J. Gildea, D. Sokaras, T.C.Weng, B. Lassalle-Kaiser, R.Tran, J.Hattne, H.Laksmono, J.Hellmich, C.Glöckner, N. Echols, R.G. Sierra, D.W. Schafer, J. Sellberg, C. Kenney, R. Herbst, J. Pines, P. Hart, S. Herrmann, R.W. Grosse-Kunstleve, M.J. Latimer, A.R. Fry, M.M. Messerschmidt, A. Miahnahri, M.M. Seibert, P.H. Zwart, W.E. White, P.D. Adams, M.J. Bogan, S. Boutet, G.J. Williams, A. Zouni, J. Messinger, P. Glatzel, N.K. Sauter, V.K. Yachandra, J. Yano, U. Bergmann, Energy-dispersive X-ray emission spectroscopy using an X-ray free-electron laser in a shot-by-shot mode, Proc. Natl. Acad. Sci. U S A. 109 (2012) 19103-19107

[111] R. Mitzner, J. Rehanek, J. Kern, S. Gul, J. Hattne, T. Taguchi, R. Alonso-Mori, R. Tran, C. Weniger, H. Schröder, W. Quevedo, H. Laksmono, R.G. Sierra, G. Han, B. Lassalle-Kaiser, S. Koroidov, K. Kubicek, S. Schreck, K. Kunnus, M. Brzhezinskaya, A. Firsov, M.P. Minitti, J.J. Turner, S. Moeller, N.K. Sauter, M.J. Bogan, D. Nordlund, W.F. Schlotter, J. Messinger, A. Borovik, S. Techert, F.M. de Groot, A. Föhlisch, A. Erko, U. Bergmann, V.K. Yachandra, P. Wernet, J. Yano, L-Edge X-ray Absorption Spectroscopy of Dilute Systems Relevant to Metalloproteins Using an X-ray Free-Electron Laser, J Phys Chem Lett. 4 (2013) 3641-3647. 